\title{
Richness of Cerrado Woody Species Engaged in Ecological Restoration in the Brazilian Federal District
}

\author{
Willian Barros Gomes ${ }^{1}$ (1) 0000-0002-9832-1982 \\ Rodrigo Studart Corrêa ${ }^{1}$ (1) 0000-0002-9422-2629 \\ Alexander Paulo do Carmo Balduíno ${ }^{1}$ (잉 0000-0003-4285-2685
}

\begin{abstract}
This study has evaluated the richness of Cerrado woody species engaged in ecological restoration in the Brazilian Federal District (BFD). A survey gathered information on plant species traded by local nurseries, species recommended in restoration plans (PRADs), species effectively introduced in areas under restoration, and species present in preserved fragments of Cerrado. Results summed 566 Cerrado woody species from 80 botanical families of which 171 species were traded by local nurseries, 277 were recommended in PRADs, 190 were effectively used in restoration projects, and 434 species were sampled in fragments of native Cerrado. We found low similarity between species composition available in nurseries, recommended in PRADs, used in restoration projects and present in preserved fragments of native Cerrado. Such results indicate a poor connection between steps related to the selection of native woody species that make up initial plant communities on sites under ecological restoration.
\end{abstract}

Keywords: Cerrado, plant diversity, rehabilitation.

\section{INTRODUCTION}

The Cerrado biome houses more than 11,000 species of vascular plants and is the richest savanna in plant species in the world (Mendonça et al., 2008). This biome has undergone severe degradation from the 1960s (Rada, 2013) mainly to support agriculture, urbanization, and mining activities (Beuchle et al., 2015; Klink \& Machado, 2005; Sano et al., 2010; Spera et al., 2016). As a result, only $54 \%$ of the original area remains under natural vegetation cover (Brasil, 2015).

Huge environmental liabilities in Brazilian biomes have triggered the demand for ecological restoration plans and projects, which aim at implementing strategies to rehabilitate natural ecosystems, environmental services, and ecological sustainability on degraded sites (Chazdon, 2008). As such, restoration projects intend to recover ecological and structural characteristics of ecosystems close to the previous original conditions (Palmer et al., 2016). Many techniques have been used to achieve restoration goals, such as natural regeneration, assisted regeneration, direct seeding, nucleation, and tree plantation, which is the most common and traditional practice of ecological restoration (Reis et al., 2010).

When projects rely on tree plantation, species richness and composition are vital characteristics of plant communities that will start up ecological succession on sites under restoration (Crouzeilles et al., 2017; Rodrigues et al., 2009; Siqueira et al., 2015). However, many projects that opted for tree plantation have selected a limited number of woody species to compose initial plant communities (Barbosa et al., 2003; Brancalion et al., 2013; Corrêa et al., 2015; Durigan et al., 2010; Rodrigues et al., 2009). Failures in restoration projects have often been attributed to low species richness and low diversity of initial plant communities (Barbosa et al., 2003).

Based on such a scenario, our objectives were to evaluate the richness of Cerrado woody species that has been recommended, available, and used for ecological restoration in the Brazilian Federal District and analyze its implications.

\footnotetext{
${ }^{1}$ Universidade de Brasília (UnB), Planaltina, DF, Brasil
} 


\section{MATERIALS AND METHODS}

\subsection{Study area}

This work was developed in the Brazilian Federal District (BFD), which is located on the Brazilian Central Plateau (Oliveira \& Pompermayer, 2012). BFD's altitude ranges from 1,000 m to 1,200 m (Martins et al., 2004), local climate is Tropical Savanna (Aw, in Köppen-Geiger classification) with dry winters, rainy summers, and an annual rainfall mean of 1,500 mm (INMET, 2018). All the fourteen Cerrado phytophysiognomies occur in the BFD (Walter, 2001) and $38.0 \%$ of BFD's territory was originally covered by savanna formations, $43.2 \%$ by grassland formations, and $18.8 \%$ by forest formations, from which $5 \%$ were gallery forests (UNESCO, 2002).

\subsection{Data gathering}

Data were gathered from (1) plant nurseries, (2) restoration plans (PRADs), (3) academic works on plant species introduced in areas under restoration, and (4) species naturally present in preserved fragments of Cerrado. These four categories of sources were surveyed until sampling sufficiency was achieved for each category. Names of Cerrado woody species from 21 nurseries, 35 PRADs, 21 implemented PRADs, and 10 fragments of Cerrado were organized for this work (Figure 1). Lists of woody species recommended in PRADs were compiled at the local environmental agency library (Instituto Brasília Ambiental - IBRAM) and at the website Biblioteca Digital (IBRAM, 2018). Taxa names were updated online as per the nomenclature of the Missouri Botanical Garden (MOBOT, 2016). Botanical families were organized according to The Angiosperm Phylogeny Group et al. (2016).

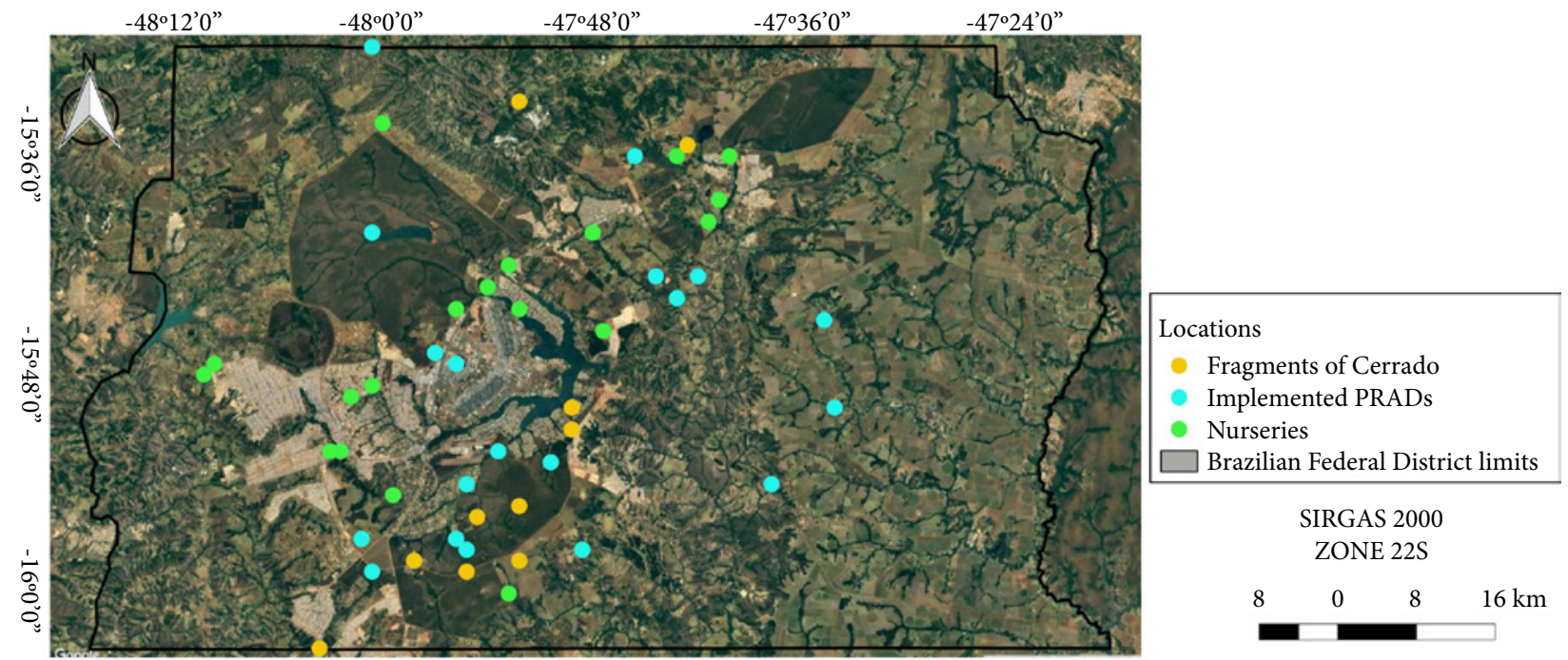

Figure 1. Location of surveyed nurseries, implemented restoration plans (PRADs) and fragments of native Cerrado in the Brazilian Federal District.

\subsection{Data analysis}

Rarefaction curves (Colwell et al., 2012) periodically tested sampling sufficiency for each of the four surveyed categories by using the software R Core Team version 3.5.1 (2017). Plant species within surveyed categories were compared by using Vegan package, according to Ugland et al. (2003), Colwell et al. (2004), and Kindt et al. (2006). Interpolated and extrapolated estimates of species richness were run at $95 \%$ confidence level as permutation allows drawing average curves of species accumulation and their empirical confidence intervals (Schilling et al., 2012). Bootsrap species richness estimator was used for species analysis and categorical data matrices were generated from the occurrence of the presence of species in each surveyed category.

Species origin was checked according to Mendonça et al. (2008) and Cerrado native species were categorized according to their natural occurrence in Cerrado phytophysiognomies (Ribeiro \& Walter, 2008). Data were organized on a table for summarizing the total number and the percentage number of Cerrado woody species engaged in each of the four surveyed categories. 


\section{RESULTS AND DISCUSSION}

The survey of Cerrado woody species in 21 nurseries, 35 PRADs, 21 implemented PRADs, and ten preserved fragments of Cerrado in the Brazilian Federal District (BFD) were enough for reaching stability tendency on rarefaction curves (Figure 2). Procedure on sampling sufficiency adopted in this study emphasizes the asymptotic response to successive samplings (Figure 2) since plant species data from tropical ecosystems do not usually achieve inflection points on rarefaction curves (Corrêa et al., 2015; Schilling et al., 2012) as also shown in our work (Figure 2).

This survey summed 566 Cerrado species from 80 botanical families, which account for $13.2 \%$ of the Cerrado's vascular plant species identified so far (Mendonça et al., 2008). There were 171 species from 45 families traded by local nurseries, 277 species from 64 families recommended in PRADs, 190 species from 52 families effectively used in restoration projects, and 434 species from 72 families were sampled in fragments from native Cerrado (Table 1).
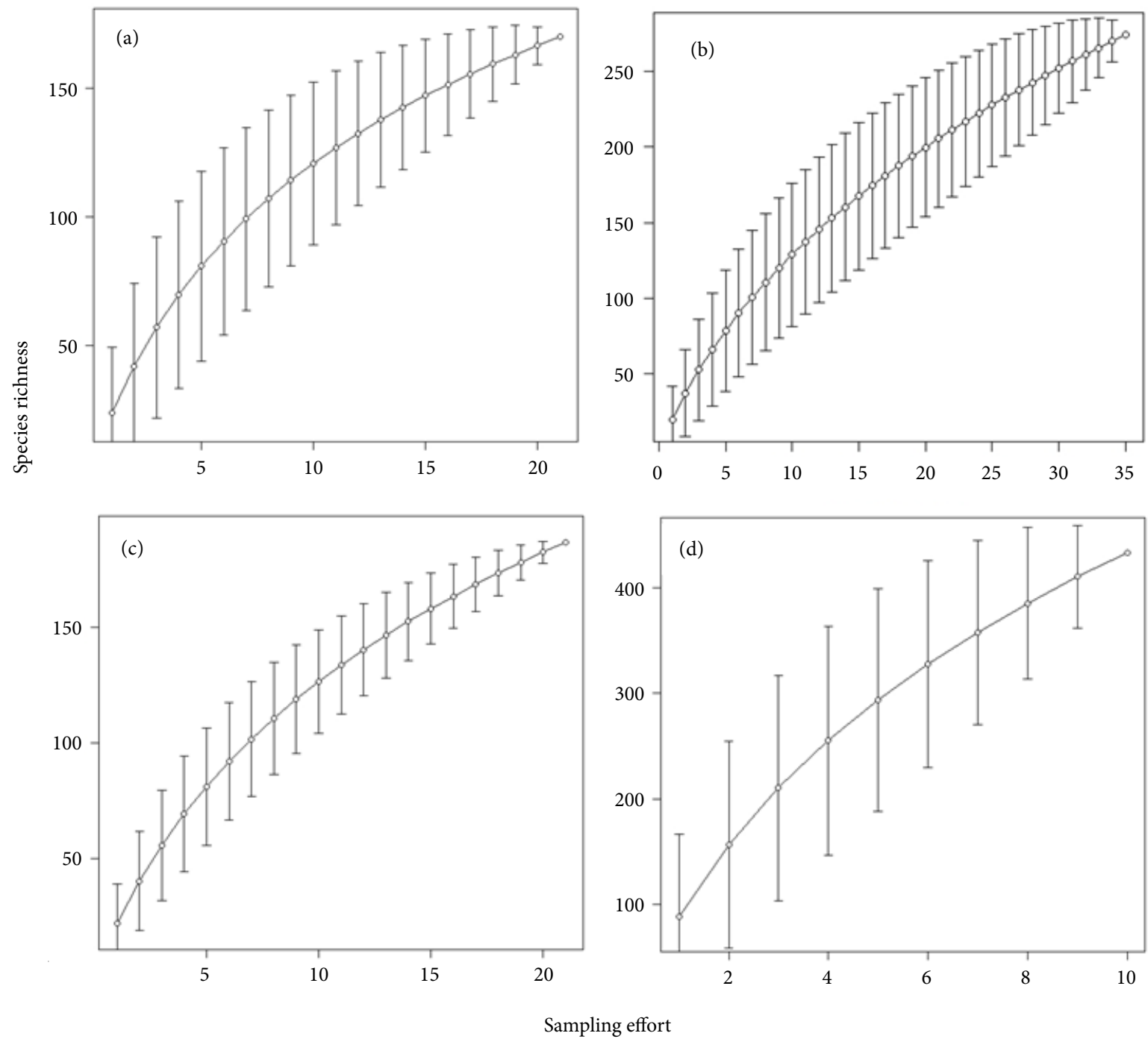

Figure 2. Rarefaction curves of Cerrado native woody species for the four surveyed categories: (a): nurseries; (b): restoration plans (PRADs); (c): implemented PRADs, and (d): preserved fragments of Cerrado. Bootstrap confidence intervals of $95 \%$ by interpolation and extrapolation. Error bars indicate estimated errors of means and unfilled dots represent sampling units. 
Table 1. Cerrado woody species available in nurseries, recommended in the restoration plans, effectively used in restoration plans, and present in preserved fragments of Cerrado in the Brazilian Federal District.

\begin{tabular}{|c|c|c|c|c|c|}
\hline Botanical family/species & $\begin{array}{c}\text { Successional } \\
\text { stage }\end{array}$ & Nursery & PRADs & $\begin{array}{l}\text { Implemented } \\
\text { PRADs }\end{array}$ & $\begin{array}{l}\text { Native } \\
\text { Cerrado }\end{array}$ \\
\hline
\end{tabular}

\section{Anacardiaceae}

Anacardium humile A. St.-Hil.*

Anacardium occidentale L.*

Astronium fraxinifolium Schott

Astronium graveolens Jacq.*

Lithraea molleoides (Vell.) Engl.

Myracrodruon urundeuva Allemão

Schinopsis brasiliensis Engl.

Schinus terebinthifolia Raddi

Spondias mombin L.*

Spondias purpurea L.

Spondias tuberosa Arruda

Tapirira guianensis Aubl.*

Tapirira obtusa (Benth.) J.D. Mitch.

Annonaceae
Annona cacans Warm.
Annona coriacea Mart.
Annona crassiflora Mart.
Annona neosericea H. Rainer
Annona tomentosa R.E. Fr.*
Cardiopetalum calophyllum Schltdl.
Duguetia furfuracea (A. St.-Hil.) Saff.*
Guatteria australis A. St.-Hil.
Guatteria sellowiana Schltdl.
Xylopia aromatica (Lam.) Mart.
Xylopia brasiliensis Spreng.
Xylopia emarginata Mart.
Xylopia sericea A. St.-Hil.
Apocynaceae

Aspidosperma cylindrocarpon Müll. Arg.

Aspidosperma discolor A. DC.

Aspidosperma eburneum Allemão ex Saldanha

Aspidosperma macrocarpon Mart.

Aspidosperma parvifolium A. DC.

Aspidosperma polyneuron Müll. Arg.

Aspidosperma pyrifolium Mart.

Aspidosperma spruceanum Benth. ex Müll. Arg.

Aspidosperma subincanum Mart. ex A. DC.

Aspidosperma tomentosum Mart.

Hancornia speciosa Gomes

Himatanthus obovatus (Müll. Arg.) Woodson*

Tabernaemontana catharinensis A. DC.

Aquifoliaceae
Ilex affinis Gardner
Ilex asperula Mart. ex Reissek
Ilex conocarpa Reissek
Araliaceae

Dendropanax cuneatus (DC.) Decne. \& Planch.

Schefflera macrocarpa (Cham. \& Schltdl.) Frodin

Schefflera morototoni (Aubl.) Maguire, Steyerm. \& Frodin

\begin{tabular}{|c|c|c|c|c|}
\hline sec. & 1 & 2 & 1 & 2 \\
\hline pio. & 5 & 3 & 2 & 2 \\
\hline sec. & 7 & 8 & 8 & 4 \\
\hline sec. & 0 & 0 & 0 & 1 \\
\hline pio. & 0 & 1 & 0 & 1 \\
\hline sec. & 10 & 7 & 9 & 1 \\
\hline pio. & 4 & 3 & 0 & 0 \\
\hline pio. & 4 & 0 & 4 & 0 \\
\hline sec. & 2 & 0 & 0 & 1 \\
\hline- & 3 & 1 & 0 & 0 \\
\hline pio. & 0 & 0 & 0 & 0 \\
\hline pio. & 6 & 10 & 6 & 1 \\
\hline sec. & 0 & 1 & 0 & 0 \\
\hline sec. & 0 & 1 & 0 & 0 \\
\hline sec. & 1 & 0 & 0 & 2 \\
\hline sec. & 3 & 10 & 2 & 4 \\
\hline sec. & 0 & 0 & 0 & 1 \\
\hline- & 0 & 0 & 0 & 1 \\
\hline sec. & 0 & 1 & 0 & 2 \\
\hline sec. & 0 & 0 & 1 & 2 \\
\hline cli. & 0 & 1 & 0 & 0 \\
\hline- & 0 & 0 & 0 & 3 \\
\hline pio. & 2 & 5 & 0 & 2 \\
\hline sec. & 0 & 0 & 0 & 2 \\
\hline sec. & 1 & 3 & 1 & 2 \\
\hline sec. & 1 & 0 & 0 & 3 \\
\hline
\end{tabular}

$\begin{array}{cllll}\text { sec. } & 0 & 0 & 0 & 1 \\ \text { sec. } & 2 & 0 & 0 & 2 \\ - & 0 & 0 & 0 & 1 \\ \text { sec. } & 4 & 5 & 2 & 5 \\ \text { sec. } & 2 & 0 & 2 & 1 \\ \text { cli. } & 3 & 1 & 0 & 0 \\ \text { sec. } & 4 & 2 & 0 & 0 \\ \text { sec. } & 2 & 0 & 0 & 2 \\ \text { sec. } & 1 & 2 & 1 & 4 \\ \text { sec. } & 2 & 5 & 1 & 4 \\ \text { sec. } & 7 & 7 & 3 & 5 \\ \text { sec. } & 0 & 2 & 1 & 1 \\ \text { pio. } & 0 & 0 & 0 & 0 \\ \text { sec. } & 0 & 0 & 0 & 1 \\ - & 0 & 1 & 0 & 0 \\ - & 0 & 0 & 0 & \end{array}$

$\begin{array}{lllll}\text { sec. } & 0 & 0 & 0 & 1 \\ \text { pio. } & 0 & 9 & 3 & 6 \\ \text { pio. } & 0 & 3 & 0 & 2\end{array}$


Table 1. Continued...

\begin{tabular}{|c|c|c|c|c|c|}
\hline Botanical family/species & $\begin{array}{l}\text { Successional } \\
\text { stage }\end{array}$ & Nursery & PRADs & $\begin{array}{l}\text { Implemented } \\
\text { PRADs }\end{array}$ & $\begin{array}{l}\text { Native } \\
\text { Cerrado }\end{array}$ \\
\hline \multicolumn{6}{|l|}{ Arecaceae } \\
\hline Acrocomia aculeata (Jacq.) Lodd. ex Mart. & pio. & 2 & 3 & 0 & 0 \\
\hline Butia capitata (Mart.) Becc. & - & 0 & 1 & 0 & 0 \\
\hline Butia purpurascens Glassman & - & 0 & 1 & 0 & 0 \\
\hline Euterpe edulis Mart. & sec. & 5 & 1 & 1 & 0 \\
\hline Mauritia flexuosa L. f.* & pio. & 6 & 1 & 0 & 0 \\
\hline Syagrus comosa (Mart.) Mart. & - & 0 & 1 & 0 & 1 \\
\hline Syagrus flexuosa (Mart.) Becc. & sec. & 0 & 0 & 0 & 2 \\
\hline Syagrus oleracea (Mart.) Becc. & sec. & 3 & 1 & 1 & 0 \\
\hline Syagrus romanzoffiana (Cham.) Glassman & sec. & 2 & 0 & 0 & 0 \\
\hline \multicolumn{6}{|l|}{ Asteraceae } \\
\hline Baccharis retusa DC. & pio. & 0 & 0 & 0 & 1 \\
\hline Chromolaena laevigata (Lam.) R.M. King \& H. Rob. & pio. & 0 & 0 & 0 & 2 \\
\hline Eremanthus capitatus (Spreng.) MacLeish & - & 0 & 1 & 0 & 0 \\
\hline Eremanthus glomerulatus Less. & - & 0 & 1 & 1 & 5 \\
\hline Eremanthus goyazensis (Gardner) Sch. Bip. & - & 0 & 0 & 0 & 2 \\
\hline Eremanthus mollis Sch. Bip. & - & 0 & 0 & 0 & 1 \\
\hline Moquiniastrum floribundum (Cabrera) G. Sancho & pio. & 0 & 0 & 0 & 1 \\
\hline Moquiniastrum polymorphum (Less.) G. Sancho & pio. & 0 & 1 & 0 & 0 \\
\hline Piptocarpha macropoda (DC.) Baker* & pio. & 0 & 0 & 0 & 3 \\
\hline Piptocarpha rotundifolia (Less.) Baker & sec. & 0 & 3 & 2 & 5 \\
\hline Vernonanthura ferruginea (Less.) H. Rob. & pio. & 0 & 0 & 0 & 1 \\
\hline Vernonanthura membranacea (Gardner) H. Rob. & - & 0 & 0 & 1 & 1 \\
\hline Vernonanthura polyanthes (Spreng.) A.J. Vega \& M. Dematt. & pio. & 0 & 1 & 0 & 0 \\
\hline \multicolumn{6}{|l|}{ Bignoniaceae } \\
\hline Cybistax antisyphilitica (Mart.) Mart.* & sec. & 9 & 2 & 7 & 3 \\
\hline Handroanthus chrysotrichus (Mart. ex A. DC.) Mattos & sec. & 5 & 1 & 2 & 1 \\
\hline Handroanthus impetiginosus (Mart. ex DC.) Mattos & sec. & 16 & 9 & 6 & 2 \\
\hline Handroanthus ochraceus (Cham.) Mattos & sec. & 7 & 8 & 5 & 5 \\
\hline Handroanthus serratifolius (Vahl) S.O. Grose & sec. & 8 & 2 & 10 & 4 \\
\hline Handroanthus umbellatus (Sond.) Mattos & sec. & 0 & 0 & 0 & 1 \\
\hline Jacaranda brasiliana (Lam.) Pers. ${ }^{*}$ & - & 1 & 2 & 1 & 1 \\
\hline Jacaranda caroba (Vell.) A. DC.* & pio. & 0 & 1 & 0 & 2 \\
\hline Jacaranda copaia (Aubl.) D. Don* & pio. & 0 & 0 & 0 & 1 \\
\hline Jacaranda cuspidifolia Mart. & pio. & 2 & 1 & 0 & 0 \\
\hline Jacaranda macrantha Cham. & pio. & 0 & 0 & 0 & 1 \\
\hline Jacaranda micrantha Cham. & sec. & 0 & 0 & 1 & 0 \\
\hline Jacaranda puberula Cham. & cli. & 0 & 0 & 0 & 1 \\
\hline Jacaranda ulei Bureau \& K. Schum. & - & 0 & 0 & 0 & 1 \\
\hline Spathodea campanulata P. Beauv.* & - & 0 & 1 & 1 & 0 \\
\hline Tabebuia aurea (Silva Manso) Benth. \& Hook. f. ex S. Moore & pio. & 8 & 3 & 5 & 3 \\
\hline Tabebuia roseoalba (Ridl.) Sandwith & pio. & 18 & 7 & 7 & 2 \\
\hline Zeyheria montana Mart. & sec. & 0 & 3 & 2 & 4 \\
\hline Zeyheria tuberculosa (Vell.) Bureau & sec. & 0 & 1 & 0 & 0 \\
\hline
\end{tabular}


Table 1. Continued...

\begin{tabular}{|c|c|c|c|c|c|}
\hline Botanical family/species & $\begin{array}{c}\text { Successional } \\
\text { stage }\end{array}$ & Nursery & PRADs & $\begin{array}{l}\text { Implemented } \\
\text { PRADs }\end{array}$ & $\begin{array}{c}\text { Native } \\
\text { Cerrado }\end{array}$ \\
\hline \multicolumn{6}{|l|}{ Bixaceae } \\
\hline Bixa orellana L.* & pio. & 1 & 1 & 0 & 0 \\
\hline Cochlospermum regium (Schrank) Pilg. & sec. & 0 & 1 & 0 & 0 \\
\hline \multicolumn{6}{|l|}{ Boraginaceae } \\
\hline Cordia sellowiana Cham.* & sec. & 0 & 1 & 1 & 2 \\
\hline Cordia trichotoma (Vell.) Arráb. ex Steud. & sec. & 1 & 0 & 1 & 1 \\
\hline \multicolumn{6}{|l|}{ Burseraceae } \\
\hline Commiphora leptophloeos (Mart.) J.B. Gillett & pio. & 0 & 2 & 0 & 0 \\
\hline Protium heptaphyllum (Aubl.) Marchand & sec. & 0 & 1 & 0 & 2 \\
\hline Protium ovatum Engl.* & sec. & 0 & 0 & 1 & 1 \\
\hline Protium spruceanum (Benth.) Engl. & sec. & 0 & 1 & 0 & 2 \\
\hline Protium unifoliolatum Engl. & - & 0 & 0 & 0 & 1 \\
\hline Tetragastris altissima (Aubl.) Swart* & - & 0 & 1 & 0 & 0 \\
\hline \multicolumn{6}{|l|}{ Calophyllaceae } \\
\hline Calophyllum brasiliense Cambess. & cli. & 7 & 11 & 8 & 2 \\
\hline Kielmeyera coriacea Mart. \& Zucc.* & sec. & 3 & 9 & 4 & 6 \\
\hline Kielmeyera lathrophyton Saddi & sec. & 0 & 0 & 0 & 2 \\
\hline Kielmeyera speciosa A. St.-Hil.. & - & 0 & 1 & 1 & 3 \\
\hline Kielmeyera variabilis Mart. \& Zucc. & sec. & 0 & 0 & 0 & 2 \\
\hline \multicolumn{6}{|l|}{ Cannabaceae } \\
\hline Celtis iguanaea (Jacq.) Sarg. & pio. & 1 & 0 & 0 & 1 \\
\hline Trema micrantha (L.) Blume & pio. & 0 & 0 & 0 & 1 \\
\hline \multicolumn{6}{|l|}{ Cardiopteridaceae } \\
\hline Citronella gongonha (Mart.) R.A. Howard & sec. & 0 & 0 & 0 & 1 \\
\hline \multicolumn{6}{|l|}{ Caricaceae } \\
\hline Jacaratia spinosa (Aubl.) A. DC.* & pio. & 1 & 0 & 0 & 0 \\
\hline \multicolumn{6}{|l|}{ Caryocaraceae } \\
\hline Caryocar brasiliense Cambess. & pio. & 10 & 17 & 3 & 6 \\
\hline \multicolumn{6}{|l|}{ Celastraceae } \\
\hline Cheiloclinium cognatum (Miers) A.C. Sm. & sec. & 2 & 1 & 0 & 3 \\
\hline Maytenus floribunda Reissek ${ }^{*}$ & sec. & 0 & 0 & 0 & 1 \\
\hline Maytenus gonoclada Mart.* & sec. & 0 & 0 & 0 & 1 \\
\hline Plenckia populnea Reissek & sec. & 0 & 1 & 2 & 5 \\
\hline Salacia crassifolia (Mart. ex Schult.) G. Don* & - & 4 & 4 & 2 & 5 \\
\hline Salacia elliptica (Mart.) G. Don* & sec. & 0 & 0 & 3 & 5 \\
\hline \multicolumn{6}{|l|}{ Chloranthaceae } \\
\hline Hedyosmum brasiliense Miq. & sec. & 0 & 1 & 0 & 2 \\
\hline \multicolumn{6}{|l|}{ Chrysobalanaceae } \\
\hline Couepia grandiflora (Mart. \& Zucc.) Benth. ex Hook. f. & sec. & 0 & 1 & 0 & 3 \\
\hline Hirtella ciliata Mart. \& Zucc. & - & 0 & 1 & 0 & 0 \\
\hline Hirtella glandulosa Spreng.* & sec. & 1 & 1 & 0 & 1 \\
\hline Hirtella gracilipes (Hook. f.) Prance ${ }^{\star}$ & sec. & 0 & 0 & 0 & 1 \\
\hline Hirtella martiana Hook. f.* & - & 0 & 0 & 0 & 1 \\
\hline Licania apetala (E. Mey.) Fritsch & - & 0 & 1 & 0 & 2 \\
\hline
\end{tabular}


Table 1. Continued...

\begin{tabular}{|c|c|c|c|c|c|}
\hline Botanical family/species & $\begin{array}{c}\text { Successional } \\
\text { stage }\end{array}$ & Nursery & PRADs & $\begin{array}{l}\text { Implemented } \\
\text { PRADs }\end{array}$ & $\begin{array}{c}\text { Native } \\
\text { Cerrado }\end{array}$ \\
\hline \multicolumn{6}{|l|}{ Chrysobalanaceae } \\
\hline Licania dealbata Hook. f. & - & 0 & 1 & 0 & 0 \\
\hline Licania octandra (Hoffmanns. ex Roem. \& Schult.) Kuntze & - & 0 & 0 & 0 & 1 \\
\hline Licania rigida Benth.* & - & 7 & 0 & 0 & 0 \\
\hline Parinari obtusifolia Hook. f. & - & 0 & 0 & 0 & 1 \\
\hline \multicolumn{6}{|l|}{ Clusiaceae } \\
\hline Clusia burchellii Engl. & - & 0 & 1 & 0 & 0 \\
\hline Clusia criuva Cambess. ${ }^{*}$ & pio. & 0 & 0 & 1 & 0 \\
\hline Garcinia brasiliensis Mart. & sec. & 1 & 0 & 0 & 1 \\
\hline Garcinia macrophylla Mart. & - & 0 & 0 & 0 & 1 \\
\hline \multicolumn{6}{|l|}{ Combretaceae } \\
\hline Buchenavia tetraphylla (Aubl.) R.A. Howard & pio. & 0 & 1 & 0 & 1 \\
\hline Buchenavia tomentosa Eichler & sec. & 4 & 2 & 4 & 0 \\
\hline Terminalia argentea Mart. & pio. & 4 & 3 & 3 & 3 \\
\hline Terminalia fagifolia Mart. & - & 0 & 0 & 1 & 3 \\
\hline Terminalia glabrescens Mart. & sec. & 0 & 2 & 0 & 2 \\
\hline Terminalia phaeocarpa Eichler & sec. & 0 & 0 & 1 & 1 \\
\hline \multicolumn{6}{|l|}{ Connaraceae } \\
\hline Connarus suberosus Planch. & sec. & 0 & 2 & 1 & 4 \\
\hline Rourea induta Planch. & sec. & 0 & 1 & 0 & 3 \\
\hline \multicolumn{6}{|l|}{ Cunoniaceae } \\
\hline Lamanonia ternata Vell. & sec. & 0 & 2 & 0 & 2 \\
\hline \multicolumn{6}{|l|}{ Dichapetalaceae } \\
\hline Tapura amazonica Poepp.* & - & 1 & 1 & 0 & 2 \\
\hline \multicolumn{6}{|l|}{ Dilleniaceae } \\
\hline Curatella americana $\mathrm{L}$. & - & 1 & 1 & 1 & 2 \\
\hline Davilla elliptica A. St.-Hil.* & - & 0 & 1 & 2 & 4 \\
\hline \multicolumn{6}{|l|}{ Ebenaceae } \\
\hline Diospyros guianensis (Aubl.) Gürke & - & 0 & 0 & 0 & 1 \\
\hline Diospyros hispida A. DC. & sec. & 0 & 3 & 2 & 7 \\
\hline Diospyros sericea A. DC. & - & 0 & 0 & 0 & 1 \\
\hline \multicolumn{6}{|l|}{ Elaeocarpaceae } \\
\hline Sloanea guianensis (Aubl.) Benth.* & sec. & 0 & 0 & 0 & 2 \\
\hline \multicolumn{6}{|l|}{ Ericaceae } \\
\hline Agarista chapadensis (Kin.-Gouv.) Judd* & - & 0 & 1 & 0 & 0 \\
\hline \multicolumn{6}{|l|}{ Erythroxylaceae } \\
\hline Erythroxylum daphnites Mart. & sec. & 0 & 1 & 0 & 3 \\
\hline Erythroxylum deciduum A. St.-Hil.* & pio. & 0 & 2 & 1 & 4 \\
\hline Erythroxylum suberosum A. St.-Hil. & sec. & 0 & 2 & 2 & 6 \\
\hline Erythroxylum tortuosum Mart. & sec. & 0 & 0 & 1 & 3 \\
\hline Erythroxylum vaccinifolium Mart. ${ }^{*}$ & pio. & 0 & 0 & 0 & 1 \\
\hline
\end{tabular}


Table 1. Continued...

\begin{tabular}{|c|c|c|c|c|c|}
\hline Botanical family/species & $\begin{array}{c}\text { Successional } \\
\text { stage }\end{array}$ & Nursery & PRADs & $\begin{array}{l}\text { Implemented } \\
\text { PRADs }\end{array}$ & $\begin{array}{l}\text { Native } \\
\text { Cerrado }\end{array}$ \\
\hline \multicolumn{6}{|l|}{ Euphorbiaceae } \\
\hline Alchornea glandulosa Poepp. & pio. & 0 & 1 & 1 & 2 \\
\hline Croton urucurana Baill. & pio. & 1 & 0 & 3 & 1 \\
\hline Mabea fistulifera Mart. & pio. & 0 & 1 & 0 & 0 \\
\hline Maprounea guianensis Aubl.* & sec. & 0 & 2 & 0 & 4 \\
\hline Sapium obovatum Klotzsch ex Müll. Arg. & - & 0 & 0 & 1 & 1 \\
\hline Sebastiania brasiliensis Spreng.* & pio. & 0 & 0 & 0 & 1 \\
\hline
\end{tabular}

\section{Fabaceae}

Acosmium lentiscifolium Schott ex Spreng.

Albizia niopoides (Spruce ex Benth.) Burkart

Albizia polycephala (Benth.) Killip

Amburana cearensis (Allemão) A.C. Sm.

Anadenanthera colubrina (Vell.) Brenan

Anadenanthera peregrina (L.) Speg.

Andira cujabensis Benth.

Andira fraxinifolia Benth.

Andira humilis Mart. ex Benth.*

Andira vermifuga Mart. ex Benth.*

Apuleia leiocarpa (Vogel) J.F. Macbr.

Bauhinia cupulata Benth.*

Bauhinia dumosa Benth.

Bauhinia forficata Link

Bauhinia longifolia (Bong.) Steud.

Bauhinia rufa (Bong.) Steud.

Bowdichia virgilioides Kunth*

Calliandra brevipes Benth.

Cassia ferruginea (Schrad.) Schrader ex DC.*

Cassia grandis L. f.

Cenostigma macrophyllum Tul.*

Centrolobium tomentosum Guillemin ex Benth.*

Chamaecrista claussenii (Benth.) H.S. Irwin \& Barneby*

Chamaecrista dentata (Vogel) H.S. Irwin \& Barneby

Chamaecrista orbiculata (Benth.) H.S. Irwin \& Barneby*

Chloroleucon tortum (Mart.) Pittier ex Barneby \& J.W. Grimes

Clitoria fairchildiana R.A. Howard

Copaifera langsdorffii Desf.*

Copaifera malmei Harms

Dalbergia densiflora Benth.

Dalbergia foliolosa Benth.

Dalbergia miscolobium Benth.

Dimorphandra mollis Benth.

Dipteryx alata Vogel

Enterolobium contortisiliquum (Vell.) Morong

\begin{tabular}{|c|c|c|c|c|}
\hline - & 0 & 1 & 0 & 0 \\
\hline sec. & 2 & 0 & 1 & 0 \\
\hline sec. & 0 & 0 & 0 & 1 \\
\hline pio. & 5 & 0 & 3 & 0 \\
\hline sec. & 6 & 8 & 8 & 2 \\
\hline sec. & 2 & 3 & 4 & 2 \\
\hline- & 0 & 1 & 0 & 0 \\
\hline sec. & 0 & 0 & 1 & 1 \\
\hline sec. & 0 & 1 & 0 & 0 \\
\hline- & 0 & 2 & 1 & 3 \\
\hline sec. & 2 & 2 & 0 & 2 \\
\hline- & 0 & 1 & 0 & 0 \\
\hline- & 0 & 0 & 0 & 1 \\
\hline sec. & 0 & 1 & 1 & 0 \\
\hline pio. & 0 & 0 & 0 & 1 \\
\hline- & 0 & 2 & 0 & 2 \\
\hline sec. & 3 & 7 & 2 & 4 \\
\hline- & 0 & 0 & 1 & 0 \\
\hline sec. & 0 & 0 & 0 & 0 \\
\hline pio. & 1 & 0 & 0 & 0 \\
\hline- & 0 & 1 & 0 & 1 \\
\hline sec. & 1 & 0 & 0 & 1 \\
\hline- & 0 & 0 & 1 & 0 \\
\hline- & 0 & 1 & 0 & 0 \\
\hline- & 0 & 0 & 2 & 1 \\
\hline pio. & 1 & 0 & 0 & 0 \\
\hline - & 1 & 0 & 0 & 0 \\
\hline sec. & 13 & 14 & 13 & 4 \\
\hline- & 0 & 1 & 0 & 0 \\
\hline- & 1 & 0 & 0 & 1 \\
\hline- & 1 & 0 & 0 & 1 \\
\hline pio. & 2 & 12 & 9 & 5 \\
\hline sec. & 1 & 9 & 0 & 5 \\
\hline sec. & 7 & 9 & 7 & 0 \\
\hline sec. & 7 & 7 & 4 & 2 \\
\hline
\end{tabular}


Table 1. Continued...

\begin{tabular}{|c|c|c|c|c|c|}
\hline Botanical family/species & $\begin{array}{c}\text { Successional } \\
\text { stage }\end{array}$ & Nursery & PRADs & $\begin{array}{l}\text { Implemented } \\
\text { PRADs }\end{array}$ & $\begin{array}{c}\text { Native } \\
\text { Cerrado }\end{array}$ \\
\hline \multicolumn{6}{|l|}{ Fabaceae } \\
\hline Enterolobium gummiferum (Mart.) J.F. Macbr. & sec. & 0 & 6 & 5 & 5 \\
\hline Enterolobium schomburgkii (Benth.) Benth & - & 0 & 2 & 0 & 0 \\
\hline Erythrina crista-galli L.* & pio. & 0 & 1 & 0 & 0 \\
\hline Erythrina fusca Lour.* & - & 0 & 0 & 1 & 0 \\
\hline Erythrina speciosa Andrews & sec. & 0 & 1 & 2 & 0 \\
\hline Erythrina velutina Willd. & pio. & 2 & 0 & 0 & 0 \\
\hline Holocalyx balansae Micheli & sec. & 1 & 0 & 0 & 0 \\
\hline Hymenaea courbaril L. & sec. & 5 & 8 & 7 & 3 \\
\hline Hymenaea martiana Hayne & - & 0 & 1 & 0 & 0 \\
\hline Hymenaea stigonocarpa Mart. ex Hayne & sec. & 6 & 11 & 8 & 4 \\
\hline Hymenolobium heringeranum Rizzini & - & 1 & 0 & 0 & 2 \\
\hline Inga alba (Sw.) Willd. & sec. & 3 & 1 & 0 & 2 \\
\hline Inga cylindrica (Vell.) Mart. & sec. & 1 & 3 & 5 & 1 \\
\hline Inga edulis Mart. & sec. & 2 & 2 & 3 & 0 \\
\hline Inga ingoides (Rich.) Willd.* & - & 0 & 0 & 0 & 1 \\
\hline Inga lateriflora Miq. & - & 0 & 1 & 0 & 0 \\
\hline Inga laurina (Sw.) Willd. & pio. & 4 & 1 & 3 & 1 \\
\hline Inga marginata Willd. & pio. & 2 & 1 & 1 & 1 \\
\hline Inga nobilis Willd. & - & 0 & 2 & 1 & 1 \\
\hline Inga sessilis (Vell.) Mart.* & sec. & 1 & 0 & 0 & 0 \\
\hline Inga vera Willd. & sec. & 4 & 2 & 1 & 0 \\
\hline Leptolobium dasycarpum Vogel & sec. & 2 & 3 & 2 & 6 \\
\hline Leptolobium elegans Vogel & sec. & 0 & 3 & 0 & 1 \\
\hline Lonchocarpus cultratus (Vell.) A.M.G. Azevedo \& H.C. Lima & sec. & 1 & 0 & 0 & 1 \\
\hline Luetzelburgia auriculata (Allemão) Ducke & - & 0 & 1 & 0 & 0 \\
\hline Machaerium acutifolium Vogel $^{\star}$ & sec. & 0 & 0 & 0 & 5 \\
\hline Machaerium amplum Benth. & - & 0 & 0 & 0 & 1 \\
\hline Machaerium nyctitans (Vell.) Benth. & pio. & 0 & 0 & 1 & 0 \\
\hline Machaerium opacum Vogel & - & 0 & 7 & 4 & 4 \\
\hline Martiodendron mediterraneum (Mart. ex Benth.) R.C. Koeppen & - & 0 & 1 & 0 & 1 \\
\hline Mimosa adenotricha Benth. & - & 0 & 1 & 0 & 0 \\
\hline Mimosa albolanata Taub. & - & 0 & 0 & 0 & 1 \\
\hline Mimosa bimucronata (DC.) Kuntze & pio. & 0 & 0 & 1 & 0 \\
\hline Mimosa caesalpiniifolia Benth. & pio. & 0 & 1 & 1 & 1 \\
\hline Mimosa claussenii Benth.* & - & 0 & 2 & 3 & 2 \\
\hline Mimosa foliolosa Benth. & - & 0 & 0 & 0 & 0 \\
\hline Mimosa heringeri Barneby & - & 0 & 0 & 0 & 1 \\
\hline Myroxylon peruiferum L. f. & sec. & 3 & 0 & 3 & 1 \\
\hline Ormosia arborea (Vell.) Harms & sec. & 2 & 0 & 0 & 0 \\
\hline Parkia pendula (Willd.) Benth. ex Walp. & sec. & 3 & 0 & 0 & 0 \\
\hline Parkia platycephala Benth. & pio. & 0 & 1 & 0 & 1 \\
\hline Peltophorum dubium (Spreng.) Taub. & sec. & 1 & 2 & 3 & 0 \\
\hline
\end{tabular}


Table 1. Continued...

\section{Botanical family/species}

Successional

Fabaceae

Piptadenia gonoacantha (Mart.) J.F. Macbr.

Piptadenia viridiflora (Kunth) Benth.

Plathymenia reticulata Benth.

Platycyamus regnellii Benth.

Platymiscium floribundum Vogel

Platypodium elegans Vogel

Poecilanthe parviflora Benth.

Poecilanthe subcordata Benth.

Poincianella pluviosa (DC.) L.P. Queiroz

Pterocarpus rohrii Vahl

Pterodon abruptus (Moric.) Benth.

Pterodon emarginatus Vogel

Pterogyne nitens Tul.

Senegalia polyphylla (DC.) Britton

Senegalia tenuifolia (L.) Britton \& Rose

Senna alata (L.) Roxb.

Senna macranthera (DC. ex Collad.) H.S. Irwin \& Barneby*

Senna multijuga (Rich.) H.S. Irwin \& Barneby

Senna pendula (Humb. \& Bonpl. ex Willd.) H.S. Irwin \& Barneby

Senna rugosa (G. Don) H.S. Irwin \& Barneby

Stryphnodendron adstringens (Mart.) Coville*

Swartzia apetala Raddi

Swartzia macrostachya Benth.

Tachigali aurea Tul.

Tachigali guianensis (Benth.) Zarucchi \& Herend.*

Tachigali rubiginosa (Mart. Ex Tul.) Oliveira-Filho

Tachigali subvelutina (Benth.) Oliveira-Filho

Tachigali vulgaris L.F. Gomes da Silva \& H.C. Lima*

Vatairea macrocarpa (Benth.) Ducke

Zollernia ilicifolia (Brongn.) Vogel

\section{Humiriaceae}

Humiria balsamifera Aubl.

Sacoglottis guianensis Benth.

Sacoglottis mattogrossensis Malme

\section{Hypericaceae}

Vismia gracilis Hieron.

Vismia guianensis (Aubl.) Pers.

\section{Lacistemataceae}

Lacistema hasslerianum Chodat*

Lamiaceae

Aegiphila integrifolia (Jacq.) B.D. Jacks.

Aegiphila verticillata Vell.*

Hyptidendron canum (Pohl ex Benth.) Harley

Vitex polygama Cham.* stage

pio

pio.

sec.

sec.

sec.

sec.

cli.

sec.

$-$

sec.

-

pio.

sec.

pio.

$$
-
$$

pio.

pio.

sec.

- 0

pio.

sec.$$
\text { - }
$$$$
\text { - }
$$

sec.
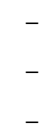

sec.

sec.

sec.

Nursery 
Table 1. Continued...

\begin{tabular}{|c|c|c|c|c|c|}
\hline Botanical family/species & $\begin{array}{c}\text { Successional } \\
\text { stage }\end{array}$ & Nursery & PRADs & $\begin{array}{l}\text { Implemented } \\
\text { PRADs }\end{array}$ & $\begin{array}{c}\text { Native } \\
\text { Cerrado }\end{array}$ \\
\hline \multicolumn{6}{|l|}{ Lauraceae } \\
\hline Aniba heringeri Vattimo-Gil ${ }^{*}$ & sec. & 0 & 0 & 0 & 1 \\
\hline Cryptocarya aschersoniana $\mathrm{Mez}^{*}$ & sec. & 0 & 0 & 0 & 2 \\
\hline Endlicheria paniculata (Spreng.) J.F. Macbr.* & sec. & 0 & 0 & 0 & 2 \\
\hline Licaria armeniaca (Nees) Kosterm. & sec. & 0 & 0 & 0 & 1 \\
\hline Nectandra cissiflora Nees & sec. & 0 & 0 & 0 & 1 \\
\hline Nectandra gardneri Meisn. & - & 0 & 0 & 0 & 1 \\
\hline Nectandra lanceolata Nees \& Mart. & sec. & 1 & 0 & 0 & 0 \\
\hline Nectandra nitidula Nees \& Mart. & sec. & 0 & 1 & 0 & 0 \\
\hline Nectandra reticulata (Ruiz \& Pav.) Mez & sec. & 0 & 0 & 0 & 3 \\
\hline Ocotea aciphylla (Nees \& Mart.) Mez & sec. & 0 & 0 & 0 & 1 \\
\hline Ocotea corymbosa (Meisn.) Mez ${ }^{\star}$ & sec. & 0 & 0 & 0 & 2 \\
\hline Ocotea densiflora (Meisn.) Mez & - & 0 & 0 & 0 & 1 \\
\hline Ocotea diospyrifolia (Meisn.) Mez & sec. & 0 & 0 & 0 & 1 \\
\hline Ocotea glaziovii Mez & sec. & 0 & 0 & 0 & 1 \\
\hline Ocotea pomaderroides (Meisn.) Mez & - & 0 & 1 & 0 & 2 \\
\hline Ocotea pulchella (Nees \& Mart.) Mez ${ }^{*}$ & cli. & 2 & 1 & 0 & 1 \\
\hline Ocotea spixiana (Nees) Mez & sec. & 0 & 0 & 0 & 3 \\
\hline Ocotea velloziana (Meisn.) Mez & sec. & 0 & 0 & 0 & 1 \\
\hline Persea fusca $\mathrm{Mez}^{*}$ & - & 0 & 0 & 0 & 2 \\
\hline \multicolumn{6}{|l|}{ Lecythidaceae } \\
\hline Cariniana estrellensis (Raddi) Kuntze ${ }^{*}$ & sec. & 4 & 2 & 3 & 2 \\
\hline Lecythis brancoensis (R. Knuth) S.A. Mori & - & 0 & 1 & 0 & 0 \\
\hline \multicolumn{6}{|l|}{ Loganiaceae } \\
\hline Antonia ovata Pohl & sec. & 0 & 0 & 0 & 1 \\
\hline Strychnos pseudoquina A. St.-Hil. & sec. & 1 & 2 & 1 & 5 \\
\hline \multicolumn{6}{|l|}{ Lythraceae } \\
\hline Diplusodon virgatus Pohl & sec. & 0 & 1 & 0 & 1 \\
\hline Lafoensia glyptocarpa Koehne & sec. & 1 & 0 & 0 & 0 \\
\hline Lafoensia pacari A. St.-Hil. & sec. & 2 & 3 & 2 & 5 \\
\hline Physocalymma scaberrimum Pohl & pio. & 3 & 1 & 2 & 0 \\
\hline \multicolumn{6}{|l|}{ Magnoliaceae } \\
\hline Magnolia ovata (A. St.-Hil.) Spreng. & sec. & 4 & 0 & 0 & 2 \\
\hline \multicolumn{6}{|l|}{ Malpighiaceae } \\
\hline Banisteriopsis megaphylla (A. Juss.) B. Gates ${ }^{*}$ & - & 0 & 0 & 0 & 1 \\
\hline Banisteriopsis stellaris (Griseb.) B. Gates & - & 0 & 0 & 1 & 1 \\
\hline Byrsonima coccolobifolia Kunth & sec. & 0 & 2 & 2 & 5 \\
\hline Byrsonima crassifolia (L.) Kunth* & pio. & 1 & 2 & 1 & 2 \\
\hline Byrsonima guilleminiana A. Juss.* & - & 0 & 0 & 0 & 1 \\
\hline Byrsonima intermedia A. Juss. & pio. & 0 & 0 & 0 & 3 \\
\hline Byrsonima laxiflora Griseb. & sec. & 0 & 1 & 0 & 2 \\
\hline Byrsonima ligustrifolia A. Juss. ${ }^{*}$ & cli. & 0 & 0 & 0 & 1 \\
\hline Byrsonima pachyphylla A. Juss. & - & 0 & 3 & 1 & 3 \\
\hline Byrsonima rotunda Griseb.* & - & 0 & 0 & 0 & 1 \\
\hline
\end{tabular}


Table 1. Continued...

\begin{tabular}{|c|c|c|c|c|c|}
\hline Botanical family/species & $\begin{array}{c}\text { Successional } \\
\text { stage }\end{array}$ & Nursery & PRADs & $\begin{array}{l}\text { Implemented } \\
\text { PRADs }\end{array}$ & $\begin{array}{c}\text { Native } \\
\text { Cerrado }\end{array}$ \\
\hline \multicolumn{6}{|l|}{ Malpighiaceae } \\
\hline Byrsonima sericea DC.* & sec. & 0 & 0 & 0 & 1 \\
\hline Byrsonima umbellata Mart. ex A. Juss.* & sec. & 0 & 0 & 0 & 1 \\
\hline Byrsonima verbascifolia (L.) DC. & sec. & 1 & 1 & 1 & 5 \\
\hline Heteropterys pteropetala A. Juss. & - & 0 & 0 & 0 & 1 \\
\hline Peixotoa reticulata Griseb. & sec. & 0 & 0 & 0 & 2 \\
\hline \multicolumn{6}{|l|}{ Malvaceae } \\
\hline Apeiba tibourbou Aubl. & pio. & 0 & 0 & 0 & 2 \\
\hline Basiloxylon brasiliensis (Allemão) K. Schum. & - & 2 & 0 & 0 & 0 \\
\hline Ceiba pentandra (L.) Gaertn. & pio. & 1 & 0 & 0 & 0 \\
\hline Ceiba pubiflora (A. St.-Hil.) K. Schum. & sec. & 0 & 2 & 0 & 1 \\
\hline Ceiba speciosa (A. St.-Hil.) Ravenna & sec. & 3 & 5 & 6 & 0 \\
\hline Eriotheca candolleana (K. Schum.) A. Robyns & sec. & 0 & 0 & 0 & 1 \\
\hline Eriotheca globosa (Aubl.) A. Robyns & - & 0 & 1 & 0 & 0 \\
\hline Eriotheca gracilipes (K. Schum.) A. Robyns ${ }^{*}$ & sec. & 0 & 0 & 1 & 2 \\
\hline Eriotheca pubescens (Mart. \& Zucc.) Schott \& Endl.* & sec. & 6 & 11 & 5 & 6 \\
\hline Guazuma crinita Mart.* & pio. & 0 & 1 & 0 & 0 \\
\hline Guazuma ulmifolia Lam. & pio. & 7 & 3 & 5 & 1 \\
\hline Luehea candicans Mart.* & sec. & 0 & 1 & 0 & 1 \\
\hline Luehea divaricata Mart. & sec. & 0 & 1 & 2 & 1 \\
\hline Luehea grandiflora Mart. & pio. & 0 & 0 & 1 & 1 \\
\hline Luehea paniculata Mart. & pio. & 1 & 0 & 0 & 2 \\
\hline Pseudobombax grandiflorum (Cav.) A. Robyns & pio. & 2 & 1 & 0 & 0 \\
\hline Pseudobombax longiflorum (Mart.) A. Robyns & sec. & 3 & 2 & 0 & 3 \\
\hline $\begin{array}{l}\text { Pseudobombax marginatum (A. St.-Hil., Juss. \& Cambess.) } \\
\text { A. Robyns }\end{array}$ & sec. & 0 & 0 & 0 & 1 \\
\hline Pseudobombax tomentosum (Mart.) Robyns ${ }^{*}$ & sec. & 0 & 1 & 3 & 2 \\
\hline Sterculia apetala (Jacq.) H. Karst. & sec. & 1 & 0 & 1 & 0 \\
\hline Sterculia striata A. St.-Hil. \& Naudin & pio. & 6 & 3 & 6 & 1 \\
\hline
\end{tabular}

\section{Melastomataceae}

Leandra aurea (Cham.) Cogn.

Leandra melastomoides Raddi

Macairea radula (Bonpl.) DC.

Miconia albicans (Sw.) Steud.

Miconia burchellii Triana*

Miconia chamissois Naudin

Miconia chartacea Triana

Miconia cubatanensis Hoehne

Miconia cuspidata Mart. ex Naudin

Miconia dodecandra Cogn.*

Miconia elegans Cogn.*

Miconia fallax DC.

Miconia ferruginata DC.*

Miconia hirtella Cogn.*

$\begin{array}{cllll}\text { sec. } & 0 & 0 & 0 & 1 \\ \text { sec. } & 0 & 0 & 0 & 1 \\ - & 0 & 1 & 0 & 0 \\ \text { pio. } & 1 & 1 & 1 & 3 \\ - & 0 & 2 & 0 & 4 \\ \text { pio. } & 1 & 0 & 1 & 2 \\ \text { pio. } & 0 & 0 & 0 & 1 \\ \text { pio. } & 0 & 0 & 0 & 2 \\ \text { sec. } & 0 & 0 & 0 & 3 \\ \text { sec. } & 0 & 1 & 0 & 1 \\ \text { sec. } & 0 & 0 & 0 & 1 \\ \text { sec. } & 0 & 0 & 0 & 1 \\ \text { pio. } & 0 & 1 & 0 & 6 \\ - & 0 & 0 & 0 & 1\end{array}$


Table 1. Continued...

\begin{tabular}{|c|c|c|c|c|c|}
\hline Botanical family/species & $\begin{array}{c}\text { Successional } \\
\text { stage }\end{array}$ & Nursery & PRADs & $\begin{array}{l}\text { Implemented } \\
\text { PRADs }\end{array}$ & $\begin{array}{c}\text { Native } \\
\text { Cerrado }\end{array}$ \\
\hline Miconia ibaguensis (Bonpl.) Triana & pio. & 1 & 0 & 1 & 0 \\
\hline Miconia leucocarpa DC. & - & 0 & 0 & 1 & 6 \\
\hline Miconia nervosa (Sm.) Triana & - & 0 & 0 & 0 & 1 \\
\hline Miconia pepericarpa DC.. & pio. & 0 & 0 & 0 & 2 \\
\hline Miconia prasina (Sw.) DC. & pio. & 0 & 0 & 0 & 1 \\
\hline Miconia punctata (Desr.) D. Don ex DC. & - & 0 & 0 & 0 & 2 \\
\hline Miconia sellowiana Naudin & pio. & 0 & 1 & 0 & 4 \\
\hline Mouriri glazioviana Cogn. & sec. & 0 & 0 & 0 & 1 \\
\hline Mouriri pusa Gardner ex Hook. & - & 0 & 1 & 0 & 0 \\
\hline Ossaea congestiflora (Naudin) Cogn. & - & 0 & 0 & 0 & 1 \\
\hline Tibouchina candolleana Cogn.* & - & 7 & 3 & 1 & 2 \\
\hline Tibouchina frigidula (DC.) Cogn.* & - & 0 & 0 & 1 & 0 \\
\hline Tibouchina granulosa (Desr.) Cogn. & sec. & 2 & 1 & 0 & 0 \\
\hline Tibouchina stenocarpa (DC.) Cogn. & - & 2 & 0 & 5 & 1 \\
\hline Tococa guianensis Aubl. & - & 0 & 0 & 1 & 0 \\
\hline Trembleya parviflora (D. Don) Cogn.* & pio. & 0 & 0 & 1 & 2 \\
\hline Trembleya phlogiformis DC. & - & 0 & 0 & 0 & 1 \\
\hline \multicolumn{6}{|l|}{ Meliaceae } \\
\hline Cabralea canjerana (Vell.) Mart.* & sec. & 0 & 1 & 1 & 2 \\
\hline Cedrela fissilis Vell. & sec. & 3 & 4 & 3 & 0 \\
\hline Cedrela odorata L. & sec. & 0 & 0 & 0 & 1 \\
\hline Guarea guidonia (L.) Sleumer & sec. & 3 & 0 & 0 & 1 \\
\hline Guarea kunthiana A. Juss.* & sec. & 0 & 0 & 1 & 1 \\
\hline Guarea macrophylla Vahl & sec. & 0 & 1 & 0 & 1 \\
\hline Trichilia catigua A. Juss.* & sec. & 0 & 0 & 0 & 1 \\
\hline Trichilia elegans A. Juss. & sec. & 0 & 0 & 0 & 1 \\
\hline Trichilia pallida Sw. & sec. & 0 & 0 & 0 & 1 \\
\hline \multicolumn{6}{|l|}{ Metteniusaceae } \\
\hline Emmotum nitens (Benth.) Miers & - & 1 & 5 & 0 & 5 \\
\hline \multicolumn{6}{|l|}{ Monimiaceae } \\
\hline Macropeplus ligustrinus (Tul.) Perkins & - & 0 & 0 & 0 & 1 \\
\hline Mollinedia oligantha Perkins ${ }^{\star}$ & - & 0 & 0 & 0 & 1 \\
\hline \multicolumn{6}{|l|}{ Moraceae } \\
\hline Brosimum gaudichaudii Trécul $^{*}$ & pio. & 2 & 3 & 2 & 3 \\
\hline Ficus citrifolia Mill. & pio. & 0 & 0 & 0 & 1 \\
\hline Ficus enormis (Mart. ex Miq.) Mart. & sec. & 0 & 0 & 0 & 1 \\
\hline Ficus insipida Willd.* & sec. & 0 & 0 & 0 & 1 \\
\hline Ficus obtusiuscula (Miq.) Miq. & sec. & 0 & 0 & 0 & 1 \\
\hline Ficus pertusa L. f. & pio. & 0 & 0 & 0 & 1 \\
\hline Ficus trigona L. f. & sec. & 0 & 0 & 0 & 1 \\
\hline Maclura tinctoria (L.) D. Don ex Steud. & sec. & 0 & 0 & 2 & 0 \\
\hline Pseudolmedia laevigata Trécul ${ }^{*}$ & sec. & 0 & 2 & 0 & 2 \\
\hline Sorocea bonplandii (Baill.) W.C. Burger, Lanj. \& Wess. Boer & sec. & 0 & 0 & 0 & 1 \\
\hline
\end{tabular}


Table 1. Continued...

\begin{tabular}{|c|c|c|c|c|c|}
\hline Botanical family/species & $\begin{array}{c}\text { Successional } \\
\text { stage }\end{array}$ & Nursery & PRADs & $\begin{array}{l}\text { Implemented } \\
\text { PRADs }\end{array}$ & $\begin{array}{c}\text { Native } \\
\text { Cerrado }\end{array}$ \\
\hline \multicolumn{6}{|l|}{ Myristicaceae } \\
\hline Virola sebifera Aubl. & pio. & 1 & 2 & 0 & 3 \\
\hline Virola urbaniana Warb. & - & 0 & 0 & 0 & 1 \\
\hline
\end{tabular}

\section{Myrtaceae}

Blepharocalyx salicifolius (Kunth) O. Berg*

Calyptranthes brasiliensis Spreng.

Calyptranthes clusiifolia (Miq.) O. Berg*

Calyptranthes lucida Mart. ex DC.

Campomanesia aromatica (Aubl.) Griseb.

Campomanesia eugenioides (Cambess.) D.Legrand ex Landrum*

Campomanesia pubescens (DC.) O. Berg

Campomanesia rufa (O. Berg) Nied.

Campomanesia velutina (Cambess.) O. Berg

Campomanesia xanthocarpa Mart. ex O. Berg*

Eugenia aurata O. Berg

Eugenia bimarginata DC.

Eugenia complicata O. Berg*

Eugenia dysenterica DC.

Eugenia florida DC.*

Eugenia involucrata DC.

Eugenia pyriformis Cambess.

Eugenia uruguayensis Cambess.

Marlierea clausseniana (O. Berg) Kiaersk.

Myrcia albotomentosa DC.

Myrcia bracteata (Rich.) DC.

Myrcia eriocalyx DC.

Myrcia fenzliana O. Berg*

Myrcia lasiantha DC.*

Myrcia nivea Cambess.

Myrcia pubipetala Miq.

Myrcia splendens (Sw.) DC.

Myrcia tomentosa (Aubl.) DC.

Myrcia venulosa DC.

Myrciaria floribunda (H. West ex Willd.) O. Berg

Myrciaria glanduliflora (Kiaersk.) Mattos \& D. Legrand

cli.

sec.

0

sec.

0

1

sec.

0

sec.

0

sec.

sec.

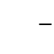

pio.

sec.

sec.

sec.

$-$

sec.

sec.

sec.

sec.

Pimenta pseudocaryophyllus (Gomes) Landrum*

Psidium firmum O. Berg*

Psidium guineense Sw.

Psidium longipetiolatum D. Legrand

Psidium myrsinites Mart. ex DC.*

Psidium myrtoides O. Berg*

Psidium oligospermum DC.*

Siphoneugena densiflora O. Berg 
Table 1. Continued...

\begin{tabular}{|c|c|c|c|c|c|}
\hline Botanical family/species & $\begin{array}{c}\text { Successional } \\
\text { stage }\end{array}$ & Nursery & PRADs & $\begin{array}{l}\text { Implemented } \\
\text { PRADs }\end{array}$ & $\begin{array}{c}\text { Native } \\
\text { Cerrado }\end{array}$ \\
\hline \multicolumn{6}{|l|}{ Nyctaginaceae } \\
\hline Guapira graciliflora (Mart. ex J.A. Schmidt) Lundell & sec. & 0 & 1 & 0 & 5 \\
\hline Guapira noxia (Netto) Lundell & sec. & 0 & 2 & 3 & 5 \\
\hline Guapira opposita (Vell.) Reitz & sec. & 0 & 0 & 0 & 1 \\
\hline Neea macrophylla Poepp. \& Endl.* & - & 0 & 0 & 0 & 1 \\
\hline Neea oppositifolia Ruiz \& Pav. & - & 0 & 0 & 0 & 1 \\
\hline Neea theifera Oerst.* & - & 0 & 3 & 2 & 2 \\
\hline \multicolumn{6}{|l|}{ Ochnaceae } \\
\hline Ouratea castaneifolia (DC.) Engl. & sec. & 0 & 1 & 0 & 2 \\
\hline Ouratea hexasperma (A. St.-Hil.) Baill. & - & 0 & 1 & 3 & 4 \\
\hline Ouratea parviflora Engl. & sec. & 0 & 1 & 0 & 1 \\
\hline \multicolumn{6}{|l|}{ Olacaceae } \\
\hline Heisteria ovata Benth. & sec. & 0 & 1 & 0 & 2 \\
\hline \multicolumn{6}{|l|}{ Oleaceae } \\
\hline Chionanthus trichotomus (Vell.) P.S. Green* & - & 0 & 0 & 0 & 1 \\
\hline \multicolumn{6}{|l|}{ Opiliaceae } \\
\hline Agonandra brasiliensis Miers ex Benth. \& Hook. f. & sec. & 0 & 1 & 0 & 3 \\
\hline \multicolumn{6}{|l|}{ Peraceae } \\
\hline Pera glabrata (Schott) Poepp. ex Baill. & pio. & 0 & 0 & 0 & 3 \\
\hline \multicolumn{6}{|l|}{ Phyllanthaceae } \\
\hline Hieronyma alchorneoides Allemão & sec. & 0 & 0 & 0 & 1 \\
\hline Margaritaria nobilis L. f.* & sec. & 0 & 0 & 1 & 1 \\
\hline Richeria grandis Vahl & sec. & 0 & 1 & 1 & 1 \\
\hline \multicolumn{6}{|l|}{ Picramniaceae } \\
\hline Picramnia sellowii Planch. & sec. & 0 & 0 & 0 & 1 \\
\hline \multicolumn{6}{|l|}{ Piperaceae } \\
\hline Piper aduncum $\mathrm{L}$. & sec. & 0 & 0 & 1 & 2 \\
\hline Piper arboreum Aubl. & - & 0 & 0 & 0 & 1 \\
\hline Piper crassinervium Kunth & sec. & 0 & 0 & 1 & 1 \\
\hline Piper hispidum Sw. & - & 0 & 0 & 0 & 1 \\
\hline Piper tectoniifolium Kunth & - & 0 & 0 & 0 & 1 \\
\hline Piper tuberculatum Jacq. & - & 0 & 0 & 1 & 0 \\
\hline \multicolumn{6}{|l|}{ Polygonaceae } \\
\hline Triplaris americana $\mathrm{L}^{*}$ & pio. & 1 & 4 & 2 & 0 \\
\hline Triplaris gardneriana Wedd. & - & 4 & 2 & 3 & 0 \\
\hline \multicolumn{6}{|l|}{ Primulaceae } \\
\hline Cybianthus detergens Mart.* & - & 0 & 1 & 0 & 3 \\
\hline Cybianthus gardneri (A. DC.) G. Agostini & - & 0 & 0 & 0 & 2 \\
\hline Cybianthus glaber A. DC. & - & 0 & 0 & 0 & 1 \\
\hline Myrsine coriacea (Sw.) R. Br. ex Roem. \& Schult. & pio. & 0 & 1 & 0 & 3 \\
\hline Myrsine gardneriana A. DC. & pio. & 0 & 0 & 1 & 1 \\
\hline Myrsine guianensis (Aubl.) Kuntze & pio. & 1 & 4 & 5 & 6 \\
\hline Myrsine lancifolia Mart. & pio. & 0 & 0 & 0 & 1 \\
\hline Myrsine umbellata Mart. & pio. & 0 & 0 & 0 & 1 \\
\hline
\end{tabular}


Table 1. Continued...

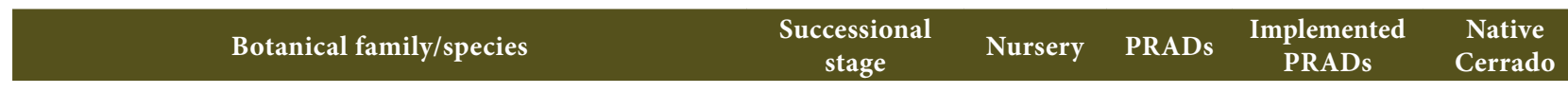

\section{Proteaceae}

Roupala montana Aubl.

sec.

1

$3 \quad 3$

7

\begin{tabular}{|c|c|c|c|c|c|}
\hline \multicolumn{6}{|l|}{ Rhamnaceae } \\
\hline Rhamnidium elaeocarpum Reissek & sec. & 1 & 0 & 1 & 1 \\
\hline
\end{tabular}

\section{Rosaceae}

Prunus brasiliensis (Cham. \& Schltdl.) D. Dietr.

Prunus chamissoana Koehne

$\begin{array}{ccccc}\text { sec. } & 0 & 0 & 0 & 1 \\ - & 0 & 0 & 0 & 1 \\ \text { cli. } & 0 & 0 & 0 & 1\end{array}$

Prunus myrtifolia (L.) Urb.

\begin{tabular}{|c|c|c|c|c|}
\hline sec. & 3 & 0 & 1 & 2 \\
\hline sec. & 0 & 0 & 0 & 3 \\
\hline- & 0 & 1 & 0 & 0 \\
\hline sec. & 0 & 0 & 0 & 1 \\
\hline- & 0 & 5 & 0 & 0 \\
\hline sec. & 0 & 0 & 0 & 1 \\
\hline sec. & 0 & 0 & 0 & 1 \\
\hline- & 0 & 0 & 0 & 1 \\
\hline sec. & 1 & 1 & 2 & 2 \\
\hline- & 0 & 0 & 0 & 0 \\
\hline sec. & 0 & 0 & 2 & 2 \\
\hline sec. & 0 & 1 & 0 & 2 \\
\hline sec. & 0 & 0 & 0 & 1 \\
\hline sec. & 0 & 1 & 0 & 2 \\
\hline- & 0 & 0 & 0 & 1 \\
\hline- & 0 & 0 & 0 & 1 \\
\hline cli. & 12 & 11 & 8 & 1 \\
\hline pio. & 0 & 0 & 0 & 1 \\
\hline sec. & 1 & 3 & 0 & 2 \\
\hline sec. & 0 & 0 & 0 & 1 \\
\hline- & 0 & 1 & 0 & 0 \\
\hline pio. & 0 & 3 & 2 & 5 \\
\hline sec. & 0 & 1 & 0 & 1 \\
\hline pio. & 0 & 0 & 0 & 1 \\
\hline sec. & 0 & 0 & 0 & 1 \\
\hline sec. & 0 & 0 & 0 & 1 \\
\hline sec. & 0 & 1 & 0 & 1 \\
\hline pio. & 2 & 1 & 0 & 5 \\
\hline
\end{tabular}

Rubiaceae

Alibertia edulis (Rich.) A. Rich. ex DC.

Amaioua guianensis Aubl.

Calycophyllum spruceanum (Benth.) Hook. f. ex K. Schum.

Chiococca alba (L.) Hitchc.

Chomelia martiana Müll.Arg.*

Chomelia obtusa Cham. \& Schltdl.

Chomelia pohliana Müll. Arg.*

Cordiera elliptica (Cham.) Kuntze

Cordiera macrophylla (K. Schum.) Kuntze

Cordiera myrciifolia (K. Schum.) C.H. Perss. \& Delprete

Cordiera sessilis (Vell.) Kuntze

Coussarea hydrangeifolia (Benth.) Müll. Arg.

Coutarea hexandra (Jacq.) K. Schum.

Faramea hyacinthina Mart.*

Ferdinandusa elliptica (Pohl) Pohl

Ferdinandusa speciosa (Pohl) Pohl

Genipa americana $\mathrm{L}$.

Guettarda pohliana Müll. Arg.

Guettarda viburnoides Cham. \& Schltdl.

Ixora brevifolia Benth.

Ladenbergia graciliflora K. Schum.

Palicourea rigida Kunth

Posoqueria latifolia (Rudge) Schult.

Psychotria carthagenensis Jacq.

Psychotria mapourioides DC.

Rudgea viburnoides (Cham.) Benth.

Rustia formosa (Cham. \& Schltdl.) Klotzsch

Tocoyena formosa (Cham. \& Schltdl.) K. Schum.

\section{Rutaceae}

Balfourodendron riedelianum (Engl.) Engl.*

Dictyoloma vandellianum A. Juss.*

Esenbeckia grandiflora Mart.

Esenbeckia pumila Pohl

Metrodorea stipularis Mart.

$\begin{array}{cllll}\text { sec. } & 1 & 0 & 0 & 0 \\ \text { pio. } & 1 & 0 & 0 & 0 \\ \text { sec. } & 0 & 0 & 0 & 1 \\ - & 0 & 0 & 1 & 1 \\ \text { sec. } & 0 & 0 & 0 & 1\end{array}$


Table 1. Continued...

\begin{tabular}{|c|c|c|c|c|c|}
\hline Botanical family/species & $\begin{array}{c}\text { Successional } \\
\text { stage }\end{array}$ & Nursery & PRADs & $\begin{array}{l}\text { Implemented } \\
\text { PRADs }\end{array}$ & $\begin{array}{l}\text { Native } \\
\text { Cerrado }\end{array}$ \\
\hline \multicolumn{6}{|l|}{ Rutaceae } \\
\hline Spiranthera odoratissima A. St.-Hil.* & - & 0 & 0 & 0 & 1 \\
\hline Zanthoxylum fagara (L.) Sarg. & sec. & 0 & 1 & 0 & 0 \\
\hline Zanthoxylum rhoifolium Lam. & sec. & 0 & 0 & 0 & 3 \\
\hline Zanthoxylum riedelianum Engl. & sec. & 1 & 2 & 0 & 1 \\
\hline \multicolumn{6}{|l|}{ Salicaceae } \\
\hline Casearia gossypiosperma Briq. & sec. & 0 & 0 & 0 & 1 \\
\hline Casearia grandiflora Cambess. & sec. & 0 & 1 & 0 & 2 \\
\hline Casearia rupestris Eichler* & pio. & 0 & 0 & 0 & 1 \\
\hline Casearia sylvestris Sw. & sec. & 1 & 1 & 2 & 5 \\
\hline Casearia lasiophylla Eichler & sec. & 0 & 0 & 0 & 1 \\
\hline Xylosma benthamii (Tul.) Triana \& Planch. & - & 0 & 0 & 0 & 2 \\
\hline Xylosma pseudosalzmanii Sleumer & sec. & 0 & 0 & 0 & 1 \\
\hline Allophylus edulis (A. St.-Hil., A. Juss. \& Cambess.) Hieron. ex Niederl. & sec. & 0 & 0 & 0 & 1 \\
\hline Cupania vernalis Cambess. & sec. & 0 & 1 & 0 & 1 \\
\hline Dilodendron bipinnatum Radlk. & pio. & 2 & 1 & 3 & 0 \\
\hline Magonia pubescens A. St.-Hil. & sec. & 3 & 2 & 4 & 1 \\
\hline Matayba elaeagnoides Radlk.* & sec. & 0 & 0 & 0 & 1 \\
\hline Matayba guianensis Aubl.* & sec. & 0 & 3 & 1 & 4 \\
\hline Sapindus saponaria L.* & sec. & 3 & 4 & 2 & 0 \\
\hline Talisia esculenta (A. St.-Hil.) Radlk. & - & 3 & 0 & 1 & 1 \\
\hline \multicolumn{6}{|l|}{ Sapotaceae } \\
\hline Chrysophyllum marginatum (Hook. \& Arn.) Radlk. & pio. & 0 & 0 & 0 & 1 \\
\hline Ecclinusa ramiflora Mart.* & sec. & 0 & 1 & 0 & 0 \\
\hline Manilkara triflora (Allemão) Monach. & - & 0 & 1 & 0 & 0 \\
\hline Micropholis venulosa (Mart. \& Eichler) Pierre & pio. & 0 & 0 & 0 & 2 \\
\hline Pouteria gardneri (Mart. \& Miq.) Baehni* & pio. & 0 & 0 & 0 & 1 \\
\hline Pouteria ramiflora (Mart.) Radlk. & sec. & 2 & 5 & 4 & 7 \\
\hline Pouteria torta (Mart.) Radlk. & sec. & 1 & 4 & 2 & 5 \\
\hline \multicolumn{6}{|l|}{ Simaroubaceae } \\
\hline Simarouba amara Aubl. & sec. & 4 & 2 & 0 & 1 \\
\hline Simarouba versicolor A. St.-Hil. & sec. & 0 & 1 & 1 & 3 \\
\hline Siparuna brasiliensis (Spreng.) A. DC. & sec. & 0 & 0 & 0 & 1 \\
\hline Siparuna guianensis Aubl. & sec. & 0 & 0 & 0 & 3 \\
\hline Solanum argenteum Dunal & - & 0 & 1 & 0 & 0 \\
\hline Solanum crinitum Lam. & sec. & 0 & 0 & 1 & 0 \\
\hline Solanum lycocarpum A. St.-Hil. & pio. & 0 & 8 & 4 & 3 \\
\hline Solanum paniculatum L. & pio. & 0 & 1 & 1 & 1 \\
\hline \multicolumn{6}{|l|}{ Styracaceae } \\
\hline Styrax camporum Pohl & sec. & 0 & 1 & 0 & 4 \\
\hline Styrax ferrugineus Nees \& Mart. & pio. & 0 & 4 & 2 & 6 \\
\hline Styrax guyanensis A. DC. & - & 0 & 1 & 0 & 1 \\
\hline Styrax pohlii A. DC. & sec. & 0 & 0 & 1 & 1 \\
\hline
\end{tabular}


Table 1. Continued...

\begin{tabular}{|c|c|c|c|c|c|}
\hline Botanical family/species & $\begin{array}{c}\text { Successional } \\
\text { stage }\end{array}$ & Nursery & PRADs & $\begin{array}{l}\text { Implemented } \\
\text { PRADs }\end{array}$ & $\begin{array}{l}\text { Native } \\
\text { Cerrado }\end{array}$ \\
\hline \multicolumn{6}{|l|}{ Symplocaceae } \\
\hline Symplocos laxiflora Benth. & - & 0 & 0 & 0 & 1 \\
\hline Symplocos nitens (Pohl) Benth. & sec. & 0 & 0 & 0 & 2 \\
\hline Symplocos revoluta Casar.* & sec. & 0 & 0 & 0 & 2 \\
\hline Symplocos rhamnifolia A. DC.* & - & 0 & 1 & 1 & 5 \\
\hline
\end{tabular}

Theaceae

Laplacea fruticosa (Schrad.) Kobuski

sec.

$\begin{array}{lll}0 & 0 & 0\end{array}$

1

\section{Thymelaeaceae \\ Urticaceae \\ Cecropia hololeuca Miq. \\ Cecropia pachystachya Trécul}

Daphnopsis fasciculata (Meisn.) Nevling*

Verbenaceae
Aloysia virgata (Ruiz \& Pav.) Pers.
Citharexylum myrianthum Cham.

\section{Vochysiaceae}

Callisthene fasciculata Mart.

Callisthene major Mart.

Qualea cordata (Mart.) Spreng.

Qualea dichotoma (Mart.) Warm.

Qualea grandiflora Mart.*

Qualea multiflora Mart.*

Qualea parviflora Mart.

Salvertia convallariodora A. St.-Hil.

Vochysia elliptica Mart.*

Vochysia haenkeana Mart.

Vochysia pruinosa Pohl

Vochysia pyramidalis Mart.

Vochysia rufa Mart.

Vochysia thyrsoidea Pohl

Vochysia tucanorum Mart.

\section{Winteraceae \\ Drimys brasiliensis Miers}

\section{Ximeniaceae}

Ximenia americana L.

Species frequency (numbers) and species successional stage: pio.: pioneer; sec.: secondary; cli.: climax. PRAD: restoration plan. ${ }^{\star}$ Woody species that naturally inhabits Cerrado grasslands formations.

The number of woody species traded by local nurseries made up $39 \%$ of the same found in native fragments of Cerrado and it may be insufficient to meet the demand of restoration plans for achieving rich and diverse plant communities. However, this scenario is better than the one found by Oliveira et al. (2017), who evaluated the availability of native species saplings in nurseries settled in the Rio Grande catchment area,
Minas Gerais (Brazil) and found a species richness lower than $10 \%$ compared to the regional native vegetation. Cerrado is the species-richest savanna in the world (Mendonça et al., 2008) and the relatively low species richness available in nurseries (39\%) is attributed to difficulties in collecting seeds from a wide range of native species and the poor knowledge on germination and growth of many native plant species 
(Oliveira et al., 2016; Oliveira et al., 2017; Santos \& Queiroz, 2011). The main obstacles for plant sapling production of Brazilian native species are seed shortage (80\%), difficulties in trading plant saplings (75\%), and poor training for the management of native species (65\%) (Silva et al., 2017).

Species richness recommended in the surveyed PRADs achieved $63.8 \%$ of that naturally present in fragments of Cerrado. However, restoration plans represent only the intention of setting up highly-diverse plant communities that will trigger ecological succession in degraded areas (Corrêa et al., 2015). Examined PRADs showed superficial and incomplete approaches to the problems intended to tackle, as some plans mostly swerved around real characteristics of sites to be restored and many proposed plant species were not adequate to them. Therefore, some PRADs were rather instruments to comply with environmental laws than to outline effective ecological restoration (Lima et al., 2006). There were lists of activities and plant species in these plans that did not match the availability of sapling species traded in BFD nurseries. Sánchez (2010) pointed out three major problems associated with PRADs: i) they usually are improperly drawn up and it results in unsatisfactory restoration when applied in practice; ii) they should be periodically updated; iii) proposed measures in PRADs are vague, generic, and difficult to check.

Studies on Cerrado phytophysiognomies have found 63 woody species in a hectare of sub-arboreal Cerrado (Cerrado stricto sensu) and 155 woody species in Cerrado's forest formations (Amaral, 2008; Andrade et al., 2002; Aquino et al., 2014; Brant, 2011; Braga \& Rezende, 2007; Haidar, 2007; Nunes et al., 2002; Silva, 2009; Silva \& Sarmento, 2009; Silva et al., 2001). Our data show local nurseries traded 26 Cerrado woody species on average, PRADs recommended 20 woody species on average, and executed PRADs used only 24 Cerrado woody species on average (Artioli, 2011; Barbosa, 2008; Carvalheira, 2007; Corrêa et al., 2007; 2015; Cortes, 2012; Ferreira et al., 2015; Fraga, 2016; Leite, 2014; Lima et al., 2016; Monteiro, 2014; Oliveira, 2013; Oliveira, 2014; Oliveira, 2015; Oliveira et al., 2015; Pachêco, 2014; Pinheiro et al., 2009; Sampaio \& Pinto, 2007; Sousa, 2016; Souza, 2002; Venturoli et al., 2013).

Low average of species richness recommended in PRADs and in executed PRADs may be a result of low availability of native species in individual nurseries, although the pool of 21 surveyed nurseries in BFD traded 171 Cerrado woody species as a whole. Thus the range of 20-24 species introduced as initial plant communities on restoration sites is not reasonable because plant species for a given PRAD can be purchased from more than one nursery. Surprisingly, we found 190 Cerrado wood species on sites where PRADs had been executed and such figure suggests that some species could have come from elsewhere besides local nurseries. The introduction of species from other populations may lead to genetic contamination, extinction of local populations, and loss of genetic biodiversity, which opposes one of the ecological restoration goals. Yet, introduction of tree saplings from distinct ecological regions brings back genes that natural selection had already banned from the receiving area or genes previously inexistent in it (Durigan et al., 2010).

Species-rich plant communities may guarantee restoration success as some studies point out that increases in ecosystem functions follow increases of species richness (Cardinale et al., 2007; Solan et al., 2009). Barbosa et al. (2003) found 355 native species in 30 plant nurseries in São Paulo State, Brazil, and an average of 30 native woody species in executed PRADs. The authors have attributed the low species richness on sites under restoration to the low availability of species in local nurseries. By comparison with our data, it seems that a low number of plant species available in individual nurseries have translated into low species richness in areas under restoration (Barbosa et al., 2003).

Qualea grandiflora Mart. was the most frequent species found in preserved fragments of Cerrado in BFD and it was present in $80 \%$ of the surveyed sites. Tabebuia roseoalba (Ridl.) Sandwith was the most frequent species available in local nurseries and it was sold by $86 \%$ of the surveyed traders. Caryocar brasiliense Cambess. was the most recommended species in PRADs and appeared listed in 49\% of them. Finally, Copaifera langsdorffii Desf. was the most frequent species effectively introduced in degraded areas and it was sampled in $62 \%$ of sites under restoration. Such a figure reflects the poor connection between the stages necessary for achieving a sound ecological restoration: reference ecosystem (Cerrado fragments), planning (PRADs), necessary support (nurseries), and execution of restoration projects.

Stepwise management of PRADs is critical for achieving successful ecological restoration (Corrêa et al., 2015). Among the 566 species recorded in this work, only 69 species (12\%) were shared in between nurseries, PRADs, executed PRADs, and Cerrado fragments. Nurseries supply plant saplings for restoration projects, and PRADs and environmental agencies cannot overlook plant species that are effectively available in local nurseries (Barbosa et al., 2003; Brancalion et al., 2013; Durigan et al., 2010; Sánchez, 2010). Approximately 37\% of the BFD territory was originally covered by sub-arboreal Cerrado (Cerrado stricto sensu) and most of the degraded sites are located in this phytophysiognomy (UNESCO, 2002). But $63.7 \%$ of species available in local nurseries, $62.5 \%$ of species recommended in PRADs, and $63.7 \%$ of species introduced on sites under restoration are from gallery forests (mata de galeria) (Table 2). 
Table 2. Percentage of Cerrado woody species and absolute number of species found in the four surveyed categories in the Brazilian Federal District, according to the phytophysiognomy of natural occurrence.

\begin{tabular}{|c|c|c|c|c|}
\hline Phytophysiognomy* & Native Cerrado & Nursery & PRADs & Implemented PRADs \\
\hline $\begin{array}{l}\text { Gallery forest } \\
\text { (mata de galeria) }\end{array}$ & $71.2 \%(309)$ & $63.7 \%(109)$ & $62.5 \%(173)$ & $63.7 \%(121)$ \\
\hline $\begin{array}{l}\text { Gallery forest } \\
\text { (mata ciliar) }\end{array}$ & $34.3 \%(149)$ & $38.0 \%(65)$ & $38.3 \%(106)$ & $36.8 \%(70)$ \\
\hline $\begin{array}{l}\text { Dry forest } \\
\text { (mata seca) }\end{array}$ & $30.2 \%(131)$ & $38.6 \%(66)$ & $29.2 \%(81)$ & $30.5 \%(58)$ \\
\hline $\begin{array}{l}\text { Arboreal Cerrado } \\
\text { (Cerradão) }\end{array}$ & $37.1 \%(161)$ & $48.5 \%(83)$ & $41.5 \%(115)$ & $41.6 \%(79)$ \\
\hline $\begin{array}{l}\text { Sub-arboreal Cerrado } \\
\text { (Cerrado stricto sensu) }\end{array}$ & $32.7 \%(142)$ & $33.9 \%(58)$ & $37.9 \%(105)$ & $41.1 \%(78)$ \\
\hline $\begin{array}{l}\text { Cerrado Park } \\
\text { (Parque de Cerrado) }\end{array}$ & $10.45(45)$ & $12.9 \%(22)$ & $12.3 \%(34)$ & $13.2 \%(25)$ \\
\hline $\begin{array}{l}\text { Palm tree formation } \\
\text { (palmeiral) }\end{array}$ & $0.7 \%(3)$ & $1.8 \%(3)$ & $1.4 \%(4)$ & $3.2 \%(6)$ \\
\hline $\begin{array}{l}\text { Grassland + palm trees } \\
\text { (Vereda) }\end{array}$ & $11.1 \%(48)$ & $9.9 \%(17)$ & $12.3 \%(34)$ & $12.1 \%(23)$ \\
\hline $\begin{array}{l}\text { Grassland } \\
\text { (campo limpo) }\end{array}$ & $4.4 \%(19)$ & $2.3 \%(4)$ & $3.2 \%(9)$ & $6.8 \%(13)$ \\
\hline Shruby grassland (campo sujo) & $15.9 \%(69)$ & $8.8 \%(15)$ & $15.5 \%(43)$ & $17.4 \%(33)$ \\
\hline $\begin{array}{l}\text { Rocky grassland } \\
\text { (campo rupestre) }\end{array}$ & $18.7 \%(81)$ & $11.1 \%(19)$ & $17.3 \%(48)$ & $24.2 \%(46)$ \\
\hline
\end{tabular}

Of the 21 implemented PRADs surveyed in this work, five $(23.8 \%)$ were executed in areas of gallery forest, six on mining sites $(28.6 \%)$, and ten $(47.6 \%)$ in areas of sub-arboreal Cerrado (Cerrado stricto sensu), which is the phytophysiognomy mostly affected by degradation in BFD (UNESCO, 2002). However, the number of plant species from sub-arboreal Cerrado (Cerrado stricto sensu) ranked the third position after gallery forest (mata de galeria) and arboreal Cerrado (Cerradão) in PRADs and implemented PRADs. Only a third of Cerrado woody species available in the surveyed nurseries are from sub-arboreal Cerrado (Cerrado stricto sensu), and it may explain the prevalence of forest species in PRADs and implemented PRADs. According to Silva et al. (2017), many species available in nurseries in Brazil are endemic and require a biome-specific approach for their use in restoration projects. Selection of native woody species for ecological restoration in BFD has shown some deficiencies, such as low species richness. Hence, implemented PRADs applied less than $40 \%$ of the species richness present in preserved fragments of Cerrado.

The low number of pioneer species in areas of executed PRADs may also be a problem as only $23.7 \%$ of plant species in such areas are pioneer species (Table 1). São Paulo State regulation SMA 32/2014 requires $40 \%$ of pioneer species to compose initial plant communities on sites that will undergo ecological restoration. Pioneer species usually grow faster than plant species of advanced ecological stages (Durigan et al., 2010) and it hastens the development of vegetation cover, which is an essential step towards the restoration of ecosystems (Corrêa et al., 2018). Another critical issue on BFD ecological restoration refers to the widespread use of forest species in areas of previously inhabited savanna formations. Such practice will likely lead succession towards the formation of forest ecosystems (Overbeck et al., 2013; Parr et al., 2014; Veldman et al., 2015).

Production of woody saplings from many different native species is a factor that currently limits ecological restoration in many parts of Brazil (Silva et al., 2017). There is currently a lack of knowledge on the production of plant saplings for several Cerrado native species (Barbosa et al., 2003; Oliveira et al., 2016; Oliveira et al., 2017; Santos \& Queiroz, 2011). As a result, it is rather difficult to find a broad sort of woody species in commercial nurseries (Oliveira et al., 2016). Seed collection and appropriate germination protocols for Cerrado species are other limitations for ecological restoration (Viani \& Rodrigues, 2007), although there are already studies on these issues (Young et al., 2005).

Besides the difficulties to produce plant saplings from Cerrado species and the low species richness in BFD nurseries and PRADs, our study shows the detachment between species composition along the line nurseries, PRADs, and executed-PRADs, as only $22.9 \%$ of species were common to these three categories. 


\section{CONCLUSION}

Cerrado woody species available in nurseries established in the Brazilian Federal District (BFD) made up 39\% of the species richness found in native fragments of Cerrado as a whole. However, species richness found on sites under restoration falls to $5.5 \%$ of it on average. Total number of plant species traded in nurseries (171) can support plant communities richer in species than the ones recommended in PRADs (20 on average) and found in areas under restoration (24 on average). Restoration plans should therefore rely on various nurseries to increase species richness in initial plant communities.

There was a higher number of Cerrado species recommended in PRADs (277) than available in BFD nurseries (171) or growing in areas of executed PRADs (190). Such a figure portraits the unrealistic nature of the surveyed restoration plans.

\section{ACKNOWLEDGEMENTS}

We would like to thank the Instituto Brasília Ambiental (IBRAM) for supporting the data collection.

\section{SUBMISSION STATUS}

Received: 17 Jan. 2018

Accepted: 18 Feb. 2019

Associate editor: João Vicente de Figueiredo Latorraca

(D)0000-0002-5969-5199

\section{CORRESPONDENCE TO}

\section{Willian Barros Gomes}

Universidade de Brasília (UnB), Área Universitária, 1, Vila Nossa Senhora de Fátima, CEP 73345-010, Planaltina, DF, Brasil

e-mail: wbgomes@yahoo.com

\section{REFERENCES}

Amaral AG. Mudanças estruturais e florísticas do estrato herbáceoarbustivo em campo sujo e campo limpo úmido na Fazenda Água Limpa - DF após um período de sete anos [thesis]. Brasília, DF: Instituto de Ciências Biológicas, Universidade de Brasília; 2008.

Andrade LAZ, Felfili JM, Violatti L. Fitossociologia de uma área de Cerrado denso na RECOR-IBGE, Brasília-DF. Acta Botanica Brasílica 2002; 16(2): 225-240. 10.1590/S0102-33062002000200009

Angiosperm Phylogeny Group; Chase MW, Christenhusz MJM, Fay MF, Byng JW, Judd WS et al. An update of the Angiosperm Phylogeny Group classification for the orders and families of flowering plants: APG IV. Botanical Journal of the Linnaean Society 2016; 181(1): 1-20. 10.1111/boj.12385

Aquino FG, Pereira CS, Passos FB, Oliveira MC. Composição florística e estrutural de um Cerrado sentido restrito na área de proteção de manancial Mestre D’Armas, Distrito Federal. Bioscience Journal 2014; 30(2): 565-575.
Artioli CG. Uso de biomantas na revegetação de um fragmento de Mata de Galeria no Jardim Botânico de Brasília, DF: sobrevivência e desenvolvimento de mudas [thesis]. Brasília, DF: Departamento de Engenharia Florestal, Universidade de Brasília; 2011.

Barbosa ACC. Recuperação de área degradada por mineração através da utilização de sementes e mudas de três espécies arbóreas do Cerrado no Distrito Federal [thesis]. Brasília, DF: Departamento de Engenharia Florestal, Universidade de Brasília; 2008.

Barbosa LM, Barbosa JM, Barbosa KC, Potomatti A, Martins SE, Asperti LM et al. Recuperação florestal com espécies nativas no estado de São Paulo: pesquisas apontam mudanças necessárias. Florestar Estatístico 2003; 6(14): 28-34.

Beuchle R, Grecchi RC, Shimabukuro YE, Seliger R, Eva HD, Sano E, Achard F. Land cover changes in the Brazilian Cerrado and Caatinga biomes from 1990 to 2010 based on a systematic remote sensing sampling approach. Applied Geography 2015; 58: 116-127. 10.1016/j.apgeog.2015.01.017

Braga FMS, Rezende AV. Dinâmica da vegetação arbórea da mata de galeria do catetinho, Brasília-DF. Cerne 2007; 13(2): 138-148.

Brancalion PHS, Lima LR, Rodrigues RR. Restauração ecológica como estratégia de resgate e conservação da biodiversidade em paisagens antrópicas tropicais. In: Peres C, Barlow J, Gardner T, Vieira IC, editors. Conservação da biodiversidade em paisagens antropizadas do Brasil. Curitiba: UFPR; 2013. p. 565-587.

Brant HSC. A fitossociologia do cerrado sentido restrito no Parque Recreativo do Gama (Prainha) - DF [monography]. Brasília, DF: Departamento de Engenharia Florestal, Universidade de Brasília; 2011.

Brasil. Mapeamento do uso e cobertura do cerrado: Projeto TerraClass Cerrado. Brasília, DF: Ministério do Meio Ambiente; 2015.

Cardinale BJ, Wright JP, Cadotte MW, Carroll IT, Hector A, Srivastava DS et al. Impacts of plant diversity on biomass production increase through time because of species complementarity. Proceedings of the National Academy of Sciences 2007; 104(46): 18123-18128. 10.1073/pnas.0709069104

Carvalheira MS. Avaliação do estabelecimento de espécies de Cerrado sentido restrito, a partir do plantio direto de sementes na recuperação de uma cascalheira na Fazenda Água Limpa - UnB [thesis]. Brasília, DF: Departamento de Engenharia Florestal, Universidade de Brasília; 2007.

Chazdon RL. Beyond deforestation: restoring forests and ecosystem services on degraded lands. Science 2008; 320(5882): 1458-1460. 10.1126/science. 1155365

Colwell RK, Chao A, Gotelli NJ, Lin SY, Mao CX, Chazdon RL, Longino JT. Models and estimators linking individual-based and sample-based rarefaction, extrapolation and comparison of assemblages. Journal of Plant Ecology 2012; 5(1): 3-21. 10.1093/jpe/rtr044

Colwell RK, Mao CX, Chang J. Interpolating, extrapolating, and comparing incidence-based species accumulation curves. Ecology 2004; 85(10): 2717-2727. 10.1890/03-0557

Corrêa RS, Balduíno APC, Teza CTV, Baptista GMM. Vegetation cover development resulting from different restoration approaches of exploited mines. Floresta e Ambiente 2018; 25(4): e20171116. 10.1590/2179-8087.111617

Corrêa RS, Melo Filho B, Baptista GMM. Avaliação fitossociológica da sucessão ecológica autogênica em áreas mineradas do Distrito Federal. Cerne 2007; 13(4): 406-415. 
Corrêa, RS, Melo Filho B, Pinheiro CQ, Santos PF. Floristic wood composition of revegetated mining sites in the Brazilian Federal District. Bioscience Journal 2015; 31(3): 908-922. 10.14393/BJv31n3a2015-22986

Cortes JM. Desenvolvimento de espécies nativas do Cerrado a partir do plantio de mudas e da regeneração natural em uma área em processo de recuperação, Planaltina, DF [thesis]. Brasília, DF: Departamento de Engenharia Florestal, Universidade de Brasília; 2012.

Crouzeilles R, Ferreira MS, Chazdon RL, Lindenmayer DB, Sansevero JB, Monteiro L et al. Ecological restoration success is higher for natural regeneration than for active restoration in tropical forests. Science Advances 2017; 3(11): e1701345. 10.1126/sciadv.1701345

Durigan G, Engel VL, Torezan JM, Melo AC, Marques M, Martins S et al. Normas jurídicas para a restauração ecológica: uma barreira a mais a dificultar o êxito das iniciativas? Revista Árvore 2010; 34(3): 471-485. 10.1590/S0100-67622010000300011

Ferreira MC, Vieira DLM, Walter BMT. Topsoil translocation for Brazilian savanna restoration: propagation of herbs, shrubs, and trees. Restoration Ecology 2015; 23(6): 723-728. 10.1111/rec.12252

Fraga LP. Efeitos da aplicação de biossólido e resíduos de poda na revegetação de área de empréstimo no Distrito Federal [thesis]. Brasília, DF: Instituto de Ciências Biológicas, Universidade de Brasília; 2016

Haidar RF. Fitossociologia, diversidade e sua relação com variáveis ambientais em florestas estacionais do bioma Cerrado no Planalto Central e Nordeste do Brasil [thesis]. Brasília, DF: Departamento de Engenharia Florestal, Universidade de Brasília; 2007.

Instituto Brasília Ambiental - IBRAM. Biblioteca Digital [Internet]. 2018 [cited 2020 Apr. 9]. Available from: https://bit.ly/2XU5MbY

Instituto Nacional de Meteorologia - INMET. Normais Climatológicas do Brasil 1981-2010 [Internet]. Brasília, DF: INMET; 2018 [cited 2020 Apr. 10]. Available from: https://bit.ly/2XRwuSK

Kindt R, Van Damme P, Simons AJ. Patterns of species richness at varying scales in western Kenya: planning for agroecosystem diversification. Biodiversity and Conservation 2006; 15(10): 32353249. 10.1007/s10531-005-0311-9

Klink CA, Machado R. Conservation of Brazilian Cerrado. Conservation Biology 2005; 19(3): 707-713. 10.1111/j.15231739.2005.00702.x

Leite TVP. Sistemas agroflorestais na recuperação de espaços protegidos por lei (AAP e Reserva Legal): estudo de caso do Sítio Geranium, DF [dissertation]. Brasília, DF: Departamento de Engenharia Florestal, Universidade de Brasília; 2014.

Lima HM, Flores JCC, Costa FL. Plano de recuperação de áreas degradadas versus plano de fechamento de mina: um estudo comparativo. REM: Revista Escola de Minas 2006; 59(4): 397-402. $10.1590 /$ S0370-44672006000400008

Lima PAF, Gatto A, Albuquerque LB, Malaquias JV, Aquino FG. Crescimento de mudas de espécies nativas na restauração ecológica de matas ripárias. Neotropical Biology and Conservation 2016; 11(2): 72-79. 10.4013/nbc.2016.112.03

Martins ES, Reatto A, Carvalho OA Jr, Guimarães RF. Evolução geomorfológica do Distrito Federal. Planaltina: Embrapa Cerrados; 2004. (Documentos; 122)
Mendonça RC, Felfili JM, Walter BMT, Silva MC Jr, Rezende AV, Filgueiras TS et al. Flora vascular do bioma Cerrado. In: Sano SM, Almeida SP, Ribeiro JF, editors. Cerrado: ecologia e flora. Brasília, DF: Embrapa Informação Tecnológica; 2008. p. 421-1279.

Missouri Botanical Garden - MOBOT. Tropicos database [Internet]. Saint Louis: MOBOT; 2017 [cited 2018 Jan. 3]. Available from: https://bit.ly/2XXi0AE

Monteiro MM. Efeito do hidrogel em plantios de mudas nativas do Cerrado para recuperação de área degradada pela mineração no Distrito Federal [thesis]. Brasília, DF: Departamento de Engenharia Florestal, Universidade de Brasília; 2014.

Nunes RV, Silva MC Jr, Felfili JM, Walter BMT. Intervalos de classe para abundância, dominância e frequência do componente lenhoso do Cerrado sentido restrito do Distrito Federal. Revista Árvore 2002; 26(2): 173-182.

Oliveira AJF. Recuperação de uma área degradada do cerrado através de modelos de nucleação, galharias e transposição de banco de sementes [dissertation]. Brasília, DF: Departamento de Engenharia Florestal, Universidade de Brasília; 2013.

Oliveira CD, Gonzaga LM, Carvalho JA, Melo LA, Davide AC, Botelho SA. Riqueza de mudas de espécies florestais nativas potencialmente produzidas na Bacia do Rio Grande, MG. Pesquisa Florestal Brasileira 2017; 37(90): 159-170. 10.4336/2017. pfb.37.90.1342

Oliveira IP, Pompermayer EF. O meio ambiente legal. Revista Faculdade Montes Belos 2012; 5(3): 1-31.

Oliveira LSC. Sucessão secundária em área de Cerrado stricto sensu durante um período de 23 anos após intervenções silviculturais [dissertation]. Brasília, DF: Departamento de Engenharia Florestal, Universidade de Brasília; 2014

Oliveira LS. Utilização de lodo de esgoto associado a três espécies nativas do Cerrado na recuperação de áreas degradadas [thesis]. Brasília, DF: Departamento de Engenharia Florestal, Universidade de Brasília; 2015.

Oliveira MC, Ribeiro JF, Passos FB, Aquino FG, Oliveira FF, Sousa SR. Crescimento de espécies nativas em um plantio de recuperação de Cerrado sentido restrito no Distrito Federal, Brasil. Revista Brasileira de Biociências 2015; 13(1): 25-32.

Oliveira MC, Ogata RS, Andrade GA, Santos DS, Souza RM, Guimarães TG et al. Manual de viveiro e produção de mudas: espécies arbóreas nativas do Cerrado. Brasília, DF: Rede de Sementes do Cerrado; 2016.

Organização das Nações Unidas para a Educação, a Ciência e a Cultura - UNESCO. Vegetação do Distrito Federal: tempo e espaço: uma avaliação multitemporal da perda de cobertura vegetal no DF e da diversidade florística da Reserva da Biosfera do Cerrado - Fase 1. 2nd ed. Brasília, DF: 2002.

Overbeck GE, Hermann JM, Andrade BO, Boldrini II, Kiehl K, Kirmer A et al. Restoration ecology in Brazil: time to step out of the forest. Natureza \& Conservação 2013; 11(1): 92-95. 10.4322/ natcon.2013.015

Pachêco BS. Chuva de sementes como indicador de restauração ecológica em matas ripárias do Distrito Federal [thesis]. Montes Claros: Centro de Ciências Biológicas e da Saúde, Universidade Estadual de Montes Claros; 2014. 
Palmer MA, Zedler JB, Falk DA. Ecological theory and restoration ecology. In: Palmer MA, Zedler JB, Falk DA, editors. Foundations of restoration ecology. 2nd ed. Washington, DC: Island Press; 2016. p. 3-26.

Parr CL, Lehmann CER, Bond WJ, Hoffmann WA, Andersen AN. Tropical grassy biomes: misunderstood, neglected, and under threat. Trends in Ecology \& Evolution 2014; 29(4): 205-213. 10.1016/j. tree.2014.02.004

Pinheiro CQ, Corrêa RS, Silveira IM, Jesus RS, Jorge RRA. Análise fitossociológica do estrato arbóreo de uma cascalheira revegetada no Distrito Federal. Cerne 2009; 15(2): 205-214.

Rada N. Assessing Brazil's Cerrado agricultural miracle. Food Policy 2013; 38: 146-155. 10.1016/j.foodpol.2012.11.002

R Core Team. R: a language and environment for statistical computing. Vienna: R Foundation for Statistical Computing; 2017 [cited 2020 Apr. 9]. Available from: http://www.R-project.org/

Reis A, Bechara FC, Tres DR. Nucleation in tropical ecological restoration. Scientia Agricola 2010; 67(2): 244-250. 10.1590/S010390162010000200018

Ribeiro JF, Walter BMT. As principais fitofisionomias do bioma Cerrado. In: Sano SM, Almeida SP, Ribeiro JP, editors. Cerrado: ecologia e flora. Brasília, DF: Embrapa Informação Tecnológica; 2008. p. 151-212.

Rodrigues RR, Lima RA, Gandolfi S, Nave AG. On the restoration of high diversity forests: 30 years of experience in the Brazilian Atlantic Forest. Biological Conservation 2009; 142: 1242-1251. 10.1016/j. biocon.2008.12.008

Sampaio JC, Pinto JRR. Critérios para avaliação do desempenho de espécies nativas lenhosas em plantios de restauração no Cerrado. Revista Brasileira de Biociências 2007; 5(S1): 504-506.

Sánchez LE. Planejamento e gestão do processo de recuperação de áreas degradadas. In: Alba JM, editor. Recuperação de áreas mineradas. 2nd ed. Brasília, DF: Embrapa Informação Tecnológica; 2010. p. 103-121.

Sano EE, Rosa R, Brito JL, Ferreira LG. Land cover mapping of the tropical savanna region in Brazil. Environmental Monitoring and Assessment 2010; 166(1-4): 113-124. 10.1007/s10661-009-0988-4

Santos JJ, Queiroz SE. Diversidade de espécies nativas arbóreas produzidas em viveiros. Enciclopédia Biosfera 2011; 7(12): 1-8.

Schilling AC, Batista JLF, Couto HZ. Ausência de estabilização da curva de acumulação de espécies em florestas tropicais. Ciência Florestal 2012; 22(1): 101-111. 10.5902/198050985083

Silva AP, Schweizer D, Marques HR, Teixeira AM, Santos TV, Sambuichi RH et al. Can current native tree seedling production and infrastructure meet an increasing forest restoration demand in Brazil? Restoration Ecology 2017; 25(4): 509-515. 10.1111/rec.12470

Silva MC Jr, Felfili JM, Walter BMT, Nogueira PE, Rezende AV, Moraes RO et al. Análise da flora arbórea de matas de galeria no Distrito Federal: 21 levantamentos. In: Ribeiro JF, Fonseca CEL, Souza-Silva JC, editors. Cerrado: caracterização e recuperação de matas de galeria. Planaltina: Embrapa Cerrados; 2001. p. 143-185.
Silva MC Jr, Sarmento TR. Comunidades lenhosas no Cerrado sentido restrito em duas posições topográficas na estação ecológica do Jardim Botânico de Brasília, DF, Brasil. Rodriguésia 2009; 60(2): 277-294. 10.1590/2175-7860200960204

Silva JS. Diversidade alfa, florística e fitossociologia na ARIE do Cerradão, na APA Gama e Cabeça de Veado, DF [thesis]. Brasília, DF: Instituto de Ciências Biológicas, Universidade de Brasília; 2009.

Siqueira G, Terra G, Garcia LC, Lima LR, Ivanaukas NM, Brienza S Jr. Ecossistemas de referência para restauração florestal. In: Brancalion PHS, Gandolfi S, Rodrigues RR, editors. Restauração florestal. São Paulo: Oficina de Textos; 2015. p. 71-102.

Solan M, Godbold JA, Symstad A, Flynn DFB, Bunker DE. Biodiversity-ecosystem function research and biodiversity futures: early bird catches the worm or a day late and a dollar short? In: Naeem S, Bunker DE, Hector A, Loreau M, Perrings C, editors. Biodiversity, ecosystem functioning and human wellbeing: an ecological and economic perspective. Oxford: Oxford University Press; 2009. p. 30-45.

Sousa AP. Avaliação de um programa de restauração de uma bacia hidrográfica: execução e envolvimento dos proprietários [thesis]. Brasília, DF: Departamento de Engenharia Florestal, Universidade de Brasília; 2016.

Souza CC. Estabelecimento e crescimento inicial de espécies florestais em plantios de recuperação de matas de galeria no Distrito Federal [thesis]. Brasília, DF: Departamento de Engenharia Florestal, Universidade de Brasília; 2002.

Spera SA, Galford GL, Coe MT, Macedo MN, Mustard JF. Land-use change affects water recycling in Brazil's last agricultural frontier. Global Change Biology 2016; 22(10): 3405-3413. 10.1111/gcb.13298

Ugland KI, Gray JS, Ellingsen KE. The species-accumulation curve and estimation of species richness. Journal of Animal Ecology 2003; 72(5): 888-897. 10.1046/j.1365-2656.2003.00748.x

Veldman JW, Overbeck GE, Negreiros D, Mahy G, Le Stradic S, Fernandes GW et al. Tyranny of trees in grassy biomes. Science 2015; 347(6221): 484-485. 10.1126/science.347.6221.484-c

Venturoli F, Venturoli S, Borges JD, Castro DS, Souza DM, Monteiro MM, Calil FN. Incremento de espécies arbóreas em plantio de recuperação de área degradada em solo de Cerrado no Distrito Federal. Bioscience Journal 2013; 29(1): 143-151.

Viani RA, Rodrigues RR. Sobrevivência em viveiro de mudas de espécies nativas retiradas da regeneração natural de remanescente florestal. Pesquisa Agropecuária Brasileira 2007; 42(8): 1067-1075. 10.1590/S0100-204X2007000800002

Walter BMT. Pesquisa botânica na vegetação do Distrito Federal, Brasil. In: Cavalcanti TB, Ramos AE, editors. Flora do Distrito Federal, Brasil. Brasília, DF: Embrapa Recursos Genéticos e Biotecnologia; 2001. p. 59-86.

Young TP, Petersen DA, Clary JJ. The ecology of restoration: historical links, emerging issues and unexplored realms. Ecology Letters 2005; 8(6): 662-673. 10.1111/j.1461-0248.2005.00764.x 